\title{
Dynamics of Continuously Pumped Solid-State Regenerative Amplifiers
}

\author{
Mikhail Grishin and Andrejus Michailovas \\ Institute of Physics \& EKSPLA uab
}

Lithuania

\section{Introduction}

Regenerative amplifiers are extensively used for amplifying pulses generated by modelocked oscillators (Koechner, 2006). This is a powerful technique providing several orders of magnitude gain, virtually unlimited by amplified spontaneous emission, (the well known nemesis for multi-pass amplifiers) (Forget et al., 2002). Such uniquely high gain is achieved due to multiple passes of optical pulse through the gain medium. Multiple passes are organized by placing the gain media in an optical resonator. The number of round trips is typically controlled by an electro-optic switch (Nickel et al., 2005) [occasionally with acousto-optic modulator (Norris, 1992)]. The optical switch also provides quality control of the optical cavity, suppressing lasing and reducing the time period when parasitic amplification of spontaneous emission takes place. In addition, the optical cavity provides "filtering" of spatial mode (there is no spatial imperfection accumulation during multiple passes of amplifying pulse). Consequently, the possibility exists to obtain perfect beam quality. At the same time the stable resonator does not allow expanding mode diameter too much (Magni, 1986), restricting capabilities for amplifying optical pulses to very high intensity - when it is required this job is placed to subsequent high aperture amplifiers (Siebold et al., 2008). An auxiliary technique, vitally important to amplify femtosecond pulses to high energies, is chirped-pulse amplification (the stretcher-compressor scheme) (Strickland \& Mourou, 1985; Mourou \& Umstadter, 1992).

In respect to the gain, the regenerative amplifiers probably have only one competitor - fiber amplifiers (Fermann et al., 2002; Liu H. et al., 2008). These amplifiers as well as all lasers based on optical fibers exhibit impressive progress over the last decade (Jeong et al., 2004). However, the extremely small mode diameter and large medium length intrinsic for optical fibers lead to significant influence of detrimental nonlinear effects. These distort the amplifying signal's optical spectrum eventually resulting in serious limitation of peak power so that $0.7 \mathrm{MW}$ before compression is one of the best achievements (Röser et al., 2007). Regenerative amplifiers are commonly designed with bulk materials allowing larger mode diameter that moves away the peak power limit well above tens of megawatts (Kleinbauer et al., 2008).

Most frequently used material for amplification of femtosecond pulses (and thoroughly dominating below $100 \mathrm{fs}$ ) is titanium doped sapphire. Broad gain bandwidth, exceptionally good lasing properties and opto-mechanical characteristics place this medium in such a special position. High pulse energy and high average output power have been achieved by

Source: Advances in Solid-State Lasers: Development and Applications, Book edited by: Mikhail Grishin,

ISBN 978-953-7619-80-0, pp. 630, February 2010, INTECH, Croatia, downloaded from SCIYO.COM 
using Ti:saphire amplifiers (Walker et al., 1999; Matsushima et al., 2006). The disadvantages of this gain medium are related to possible means of pumping. First, corresponding absorption lines are located in the green spectrum range, where suitable laser diodes with reasonable power are not available. Second, the short gain relaxation time $(3.2 \mu \mathrm{s})$ requires pulsed pumping with high pulse energy (usually with Q-switched frequency-doubled neodymium lasers) in order to store substantial population inversion and consequently to obtain high output energy. We should remark here that the issues described in present chapter are not valid for Ti:saphire regenerative amplifiers since we use approximations which suitable only for long lifetime media. From the other hand due to very short lifetime the Ti:saphire amplifiers do not exhibit extraordinary dynamic properties on which we are mainly focusing in the chapter.

Another family of popular laser materials is ytterbium doped crystals and glasses. Their wide spectrum supports amplification of ultrashort pulses (however not as short as Ti:sapphire supports). Moreover ytterbium materials allow direct laser diode pumping with intrinsically small quantum defect (typically $10 \%$ or even less). The latter enhances overall power efficiency and reduce heat generation in active elements, that in turn alleviates thermal effects which inhibit average power increase. Long upper-level lifetime of ytterbium ions virtually in all the crystals and glasses allows good capacity of stored energy under convenient continuous laser diode pumping. What somewhat challenges operation with ytterbium materials is relatively low gain (typically less than $10 \%$ per pass) which originated from peculiar to this materials small stimulated emission cross section. From the other hand regenerative amplification is indeed a way of efficient energy extraction at low gain; just special attention should be given to reduce inrtracavity losses (Biswal et. al., 1998). An alternate way to improve stimulated emission features is cryogenically cooled active elements (Kawanaka et al., 2003) although this method is bulky and, as a rule, it narrows gain bandwidth. Then special attention should be placed to host crystal selection not to limit development towards shorter pulses (Pugžlys et al., 2009).

Ytterbium doped media are able to withstand very intense optical pumping without detriment to exited-state population which in other materials can be limited by quenching effects (e.g. exited state absorption or up-conversion). This favorable property permits use of active elements in thin disc geometry. In particular, Yb:YAG thin disk lasers are scalable to very high average power and to high pulse energies (Speiser \& Giesen, 2008). Extremely short optical pass within the thin disc reduces nonlinear effects (in essence the Kerr effect) allowing high peak power pulses even without using stretcher-compressor technique (Kleinbauer et al., 2008).

The amplifiers based on neodymium gain media have their specific advantages. High stimulated emission cross section simplifies system design and reduces requirements for optical components. Good energy storage capabilities allow high output energy and power efficient operation when pumping with laser diodes. Neodymium laser materials are well suited for picosecond pulse durations and are competitive for moderate average power. Systems based on $\mathrm{Nd}: \mathrm{YVO}_{4}$ and $\mathrm{Nd}: \mathrm{GdVO}_{4}$ crystals routinely produce more than $10 \mathrm{~W}$ of output power (Kleinbauer et al., 2005; Clubley et al., 2008).

The regenerative amplifiers of solid state lasers designed for scientific applications usually operate at low or moderate repetition rates (not exceeding several $\mathrm{kHz}$ ). Presently, there is rising demand for high repetition rate ultrashort-pulse solid state lasers for material microprocessing (Meijera et al., 2002). On the other hand, a new generation of fast electro-optic 
switches became available such as Pockels cells based on $\beta$-barium borate along with improved high-voltage electronics (Nickel et al., 2005; Siebold et al., 2004). As a result, picosecond and femtosecond lasers with repetition rate of the order of 100's kHz have come onto the market (Raciukaitis et al., 2006). Regenerative amplifiers are an important part of most ultrafast industrial solid state laser systems. Both high system efficiency and stable output parameters over a wide range of pulse repetition rates are essential for this actively developing field. For creation of power-efficient systems, neodymium and ytterbium laser gain media which may be directly pumped by laser diodes is advantageous. Long lifetime of the upper laser level typical of both these ions supports accumulation of substantial population inversion under continuous laser diode pumping. However, this long inversion lifetime may also cause stability problems at high repetition rates. Continuously pumped regenerative amplifiers demonstrate peculiar pulse amplification dynamics when the pulse repetition period becomes comparable or shorter than the gain relaxation time (Müller et al., 2003). Period doubling bifurcations develop generating periodically alternating energy pulses or even sequences of pulses having chaotic energy distribution.

Complex dynamic behavior is well known phenomenon in laser physics (Haken, 1975). Generally, nonlinear differential equations describing laser dynamics tend to have unstable solutions containing multi-stabilities and bifurcations when a number of independent variables representing system states are equal or more than three (Lorenz, 1963). By no means complete list of laser systems exhibiting complicated dynamics includes Q-switched gas lasers (Arecchi et al., 1982) passively Q-switched solid state lasers (Tang et al., 2003), optically injected solid state lasers (Valling et al., 2005). The specifics of high repetition rate regenerative amplifiers is such that their operation can be described with two differential equations, but periodic disturbance caused by release of the amplified pulse complicates the system behavior. Unlike many lasers which have been created specially to study dynamic phenomena and chaotic behavior, dynamics of regenerative amplifiers needs to be understood from a more utilitarian position in order to comprehensively optimize real systems. To date, only a few articles have been dedicated to this phenomenon despite its critical influence on the performance of regenerative amplifiers. Period doubling regime passing to chaotic operation has been observed for a system based on ytterbium doped glass and the role of bifurcations has been investigated both theoretically and experimentally (Dörring et al., 2004). However, one of the important parameters, the seed pulse energy, was left beyond the scope, and so applicability of the obtained results was restricted. The experiments were confined to studying cavity dumping of the Q-switched laser, an approximately equivalent system to the regenerative amplifier seeded by extremely low pulse energy. Our recent theoretical work has presented a generalized picture of stability features of a continuously pumped high repetition rate regenerative amplifier based on laser media with long relaxation time. The regions exhibiting different system behavior have been mapped in the space of non-dimensional control parameters: repetition rate and round trip number (Grishin et al., 2007). Additionally this analysis revealed the importance of the seed pulse energy and demonstrated that increase in the seed energy helps in eliminating the instabilities. Comprehensive utilization of these theoretical results has promoted top performance obtained from multi-kilohertz Yb:YAG disk amplifier (Metzger et al., 2009). Experimental study, performed with $\mathrm{Nd}: \mathrm{YVO}_{4}$ system, has thoroughly confirmed theoretical conclusions and on its basis the concept of regenerative amplifiers optimization has been formulated (Grishin et al., 2008; Grishin et al., 2009). 
In the present chapter we summarize information from our already published papers and expand this description on the basis of our recent results. In the first theoretical part of the chapter we present a concept for modeling of continuously pumped solid state regenerative amplifiers. Simplified rate equations in normalized form allow reducing the system analysis to a task which has been thoroughly accomplished in theory of discrete-time dynamical systems (Alligood et al., 1996). Then we focus more attention to the influence of the main governing parameters on dynamics of regenerative amplification and limitations of the practical system performances occurred due to instabilities. We show why seed pulse energy plays such an important role at repetitive regime in contrast to low repetition rates. Influence of parasitic intracavity losses is shown to be a factor which not only decreases energy extraction efficiency (as in all laser systems) but also enhances instability range. We analyze numerically obtained stability diagrams which allow determination of optimal operation regime when maximum output energy can be extracted from the amplifier as stable pulse train. Important laser parameters which influence the system performance indirectly will be considered too. Heating of intracavity optical components depends not only on the range of output average power and the optical components quality but also on the amplification regime. The Kerr nonlinearity which limits system performance for short optical pulses will be evaluated taking into account multiple passes.

The verification of the created model is presented in the experimental part of the chapter. We investigate operation peculiarities of the system consisting of mode-locked master oscillator, the preamplifier and the regenerative amplifier based on $\mathrm{Nd}: \mathrm{YVO}_{4}$ crystals and analyze criteria of stable operation limitation. The experimental dependences of the output energy versus round trip number and repetition rate well agree with theoretical data. We demonstrate that increase of the seed pulse energy up to the value predicted by numerical model ensures stable operation within full range of repetition rates. The chapter is concluded by summarizing of presented theoretical and experimental results and suggesting of further investigations.

\section{Theory of regenerative amplification in repetitive regime}

\subsection{Principle of operation}

Regenerative amplifier can be regarded as a system in which an optical resonator provides multiple passes, a gain medium is responsible for amplification, and an electro-optic switch serves as a valve in-turn admitting weak input pulse and releasing amplified pulse. At that, spatial properties of the amplified radiation are primarily determined by the optical resonator; the output energy mostly depends on the population inversion stored in the gain medium whereas the amplified pulse duration is imposed by the input seed pulse. A schematic diagram of a conventional solid-state diode-pumped regenerative amplifier is depicted in Fig. 1. This optical layout by no means differs from that for a common Qswitched laser and consequently there are certain similarities in operation (Murray \& Lowdermilk, 1980). The resonator quality (Q-factor) is controlled by an electro-optic switch. The electro-optic switch usually consists of a Pockels cell, a quarter-wave plate and a polarizer. An operational cycle of the regenerative amplifier consists of two successive stages: low-Q and high-Q. When voltage is not applied to the Pockels cell the wave plate along with the polarizer provides high intracavity losses (low-Q state of the resonator). Laser action is suppressed by high losses, and the gain medium, being under continuous pumping, accumulates population inversion. The amplification takes place during high-Q 
stage. It starts when quarter-wave voltage is applied to the Pockels cell and the seed pulse is injected into the resonator. The intracavity losses become minimal and are kept low for some pre-set time while the optical pulse circulating in the resonator is amplifying, simultaneously consuming a certain part of the stored energy. The intracavity pulse energy grows until the gain becomes equal to the resonator losses and then the pulse energy decays. As soon as the intracavity energy reaches a desired level the Pockels cell voltage is switched off. This dumps the amplified pulse out of the cavity as the output pulse. The system returns into the initial state. Then during the next low-Q stage the depleted part of the inversion population is restored by uninterrupted pumping and the cycles iterate.

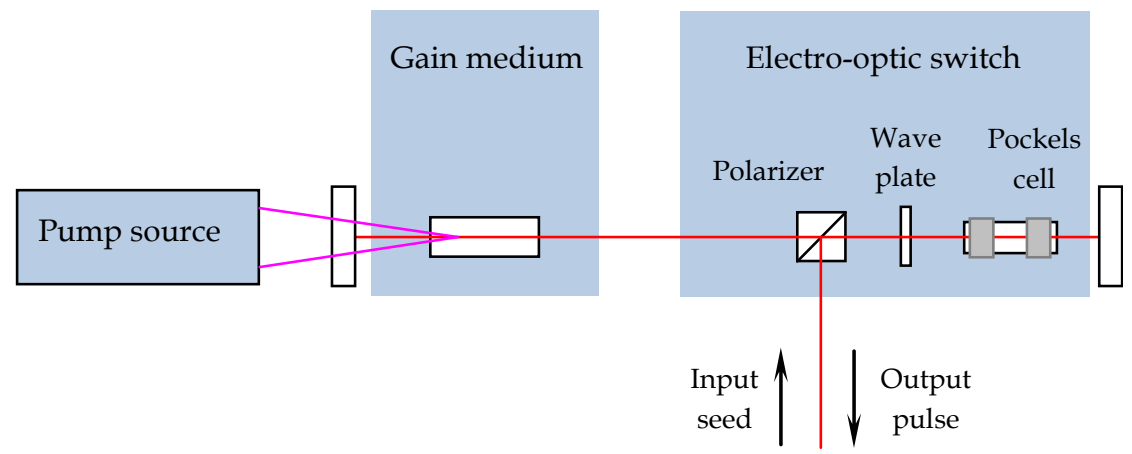

Fig. 1. Optical layout of typical solid-state diode pumped regenerative amplifier.

At higher repetition rates, when the pump stage is comparable or shorter than the gain relaxation time, the operation cycles become interdependent. The equilibrium between population inversion depletion caused by amplification and inversion restoration caused by pumping may become unstable. This often leads to breakdown of the single-energy regime and to generation of periodically alternating high/low energy pulses, or more complicated instability patterns. We will consider this and related phenomena theoretically in the first part of the present chapter.

\subsection{Rate equations and basic terms}

The process of regenerative amplification is essentially determined by the interaction of the intracavity radiation with the laser medium excited by pumping. The rules of this interaction can be established by using a simple phenomenological notion of a dynamic balance between basic processes: pumping and relaxation, amplification and extinction. Thus the system evolution can be described by coupled differential equations for the population inversion density $N$ and photon number $\phi$. We use the space independent rate equations which have been formulated for idealized four-level laser medium with a homogenously broadened line (Svelto, 1998):

$$
\begin{aligned}
& \frac{d N}{d t}=R_{p}-\frac{\sigma c}{V} \phi N-\frac{N}{T_{1}} \\
& \frac{d \phi}{d t}=\left(\frac{\sigma c L_{a} A_{a}}{V} N-\frac{1}{T_{c}}\right) \phi .
\end{aligned}
$$


The density of population inversion grows due to pumping (proportionally to the pumping rate $R_{p}$ ) and decays with the constant rate inversely proportional to the upper laser level lifetime $T_{1}$. This balance is significantly influenced by stimulated emission, the contribution of which depends on the gain medium characteristics and particularly on the stimulated emission cross section $\sigma$. The second equation establishes a rule of stimulated photons multiplication. The photon number increase proportionally to the population inversion and at the same time it decreases because of intracavity losses. The losses here are defined in terms of the photon lifetime $T_{c}$ which determines the rate of decay for the light field in the optical resonator. Geometry of the amplifier is accounted for in terms of the mode volume within the optical resonator $(V)$, the active medium length $\left(L_{a}\right)$ and the beam area in the active medium $\left(A_{a}\right)$. The velocity of light $c$ is a constant coupling temporal and spatial terms of the equations.

Since we use the rate equations approximation for modeling, the range of proposed model applicability is limited by the range of the particular rate equation's validity. Concerning general validity of the rate equation approach, we refer to (Svelto, 1998) for details. Here we just mention that this is a conventional method of laser dynamics study. It gives sufficiently accurate results for most practical purposes and in particular for analysis of multi-pass and regenerative amplification (Murray \& Lowdermilk, 1980; Lowdermilk \& Murray, 1980)

We shall re-arrange the equations to a form containing macroscopic non-dimensional terms. This is a common way to reduce the number of control parameters in order to provide simplified picture of system behavior without losing information. A primary normalization coefficient is the term proportional to the pump level, $G_{0}=R_{p} T_{1} \sigma L_{a}$. The physical meaning of $G_{0}$ is steady state gain coefficient per pass. Such gain is achieved at the equilibrium of population inversion density $(d N / d t=0)$ obtained under constant pumping $\left(d R_{p} / d t=0\right)$, constant rate of spontaneous decay $\left(T_{1}\right)$ and in absence of lasing $(\phi=0)$. Thus we can proceed to equations for the normalized gain $g=N \sigma L_{a} / G_{0}$ and normalized energy $\varepsilon=\phi \sigma /\left(A_{a} G_{0}\right)$ :

$$
\begin{gathered}
\frac{d g\left(t^{\sim}\right)}{d t^{\sim}}=-\varepsilon\left(t^{\sim}\right) g\left(t^{\sim}\right)+\frac{1-g\left(t^{\sim}\right)}{\tau_{1}} \\
\frac{d \varepsilon\left(t^{\sim}\right)}{d t^{\sim}}=\varepsilon\left(t^{\sim}\right)\left[g\left(t^{\sim}\right)-g_{t}\right] .
\end{gathered}
$$

Additionally, we have modified the time scale by introducing normalized time $t^{\sim}=t G_{0} / T_{0}$, a product of the current number of cavity round trips and the steady state gain coefficient. Here the denominator $T_{0}$ represents the round trip time. As we will see later, the term $t^{\sim}$ assigns a natural time scale to the high-Q phase of operation. The governing parameter, which defines the amplification period (effective round trip number), will be introduced on this basis. The normalized relaxation time of the gain $\tau_{1}=T_{1} G_{0} / T_{0}$ is essentially determined by the upper laser level lifetime $T_{1}$. A term accounting for parasitic losses of the optical resonator is represented by normalized threshold gain, $g_{t}=T_{0} /\left(T_{c} G_{0}\right)$. This parameter can be also expressed in terms of loss coefficient $l$ as $g_{t}=-\ln (1-l) / G_{0}$, by using common threshold criterion of gain and losses equality.

Note that equations formulated with those new variables do not contain pumping characteristics in explicit form. Parameter $G_{0}$, proportional to the pumping rate, is hidden in 
the composition of basic variables. The pump effect (as well as other control parameters effects) is easy to restore when applying modeling results by performing reciprocal transformation from normalized to real parameters. Moreover, further in the theoretical part of this chapter we often omit the words "effective" and "normalized" just for shortening. Although Eqs. (3) and (4) have been obtained in the approximation of a four-level system, the identical equations can be formulated for the more general case of quasi-three-level systems [the initial rate equations and conditions of their applicability can be found in (Svelto, 1998)]. In the latter case the explicit expressions of normalized and effective terms look slightly more complicated but their physical meaning remains unchanged.

\subsection{Consideration of a single operation cycle}

Below in this section we define relations between basic system parameters deriving the rate equations for separate stages of regenerative amplifier operation. First we consider the low$Q$ phase. Since during this phase of operation amplification is suppressed while pumping takes place, it is also called pump stage. Inasmuch as there is no lasing during this stage (i.e. $\varepsilon=0$ ), a set of Eqs. (3) and (4) transforms into a single equation:

$$
\frac{d g\left(t^{\sim}\right)}{d t^{\sim}}=\frac{1-g\left(t^{\sim}\right)}{\tau_{1}}
$$

Initial conditions specify the gain at the beginning of the pump phase, $g_{p i}$. Taking into account that $t^{\sim} / \tau_{1}=t / T_{1}$, we can find the relation between the initial gain $g_{p i}$ and the final gain $g_{p f}$ for a certain pump phase duration $T$ :

$$
g_{p f}=1-\left(1-g_{p i}\right) \exp \left(-\frac{T}{T_{1}}\right)
$$

In the next section we will often use diminished form of the equation (6): $g_{p f}=\hat{g}_{p}\left(g_{p i}, T / T_{1}\right)$, where the function $\hat{g}_{p}$ just establishes a rule of the gain transformation $g_{p i} \rightarrow g_{p f}$ for the pump stage.

Then let us proceed to the high-Q phase. The equations for this operation stage can be simplified as well. We remind here that we explore laser media having long relaxation time, the case functionally important for diode-pumped systems. Since the buildup time of the optical pulse is usually short in comparison with the pump phase duration and the upper laser level lifetime, the population inversion change due to pumping and relaxation processes is much smaller than the inversion depletion caused by amplification. Hence, the terms containing spontaneous decay and optical pumping can be neglected in Eq. (3). Also, as we have assumed negligible pump contribution during high-Q phase, this stage of operation can be called in a more common and more informative manner as the amplification stage.

The next assumption presumes low intra-cavity losses $\left(g_{t} \rightarrow 0\right)$. This approximation substantially simplifies the basics of the presented method. The simplification appears not only due to existence of the analytical solution for the rate equations but also, more importantly, because of the reduced number of parameters governing the system. An influence of the parasitic losses on amplification dynamics will be accounted in section 2.7 after the essence of the theoretical approach has been presented. For the present, we come to 
the situation at which Eqs. (3) and (4), when describing amplification stage, reduce to the following:

$$
\begin{aligned}
& \frac{d g\left(t^{\sim}\right)}{d t^{\sim}}=-\varepsilon\left(t^{\sim}\right) g\left(t^{\sim}\right) \\
& \frac{d \varepsilon\left(t^{\sim}\right)}{d t^{\sim}}=\varepsilon\left(t^{\sim}\right) g\left(t^{\sim}\right) .
\end{aligned}
$$

Initial conditions specify the system state at the beginning of the amplification phase: the initial gain $g_{a i}$ and the energy of the input pulse from which the amplification starts - the seed energy, $\varepsilon_{s}$. Also it is natural to constrain our consideration to the case of low seed energy with respect to the stored energy $\left(\varepsilon_{s}<<g_{a i}\right.$ in terms of normalized parameters). As a consequence of these assumptions, the solutions of coupled Eqs. (7) and (8) can be found in analytic form. Such solutions obviously describe temporal evolution of the gain and intracavity energy. One can also find other physical sense, more convenient for further consideration. In case of fixed amplification phase duration, temporal evolution is terminated at the moment $t^{\sim}=\tau$. Then the solutions also express how the system parameters on the amplification phase completion (final gain $g_{a f}$ and output pulse energy $\varepsilon_{f}$ ) depend on the initial conditions and governing parameters:

$$
\begin{gathered}
g\left(t^{\sim}\right)=\frac{g_{a i}}{1+\frac{\varepsilon_{s}}{g_{a i}} \exp \left(g_{a i} \tau^{\sim}\right)} \Rightarrow g_{a f}=\frac{g_{a i}}{1+\frac{\varepsilon_{s}}{g_{a i}} \exp \left(g_{a i} \tau\right)} \\
\varepsilon\left(t^{\sim}\right)=\frac{g_{a i}}{1+\frac{g_{a i}}{\varepsilon_{s}} \exp \left(-g_{a i} t^{\sim}\right)} \Rightarrow \varepsilon_{f}=\frac{g_{a i}}{1+\frac{g_{a i}}{\varepsilon_{s}} \exp \left(-g_{a i} \tau\right)} .
\end{gathered}
$$

Here the terms $\tau$ and $\varepsilon_{s}$ are actual parameters controlling the amplification, whereas the initial gain is a variable coupling the equations. The normalized amplification stage duration $\tau$ is a product of cavity round trip number and steady state gain $G_{0}$. Further we will call this term in a more comprehensible manner an "effective round trip number". Essentially, the term $\tau$ also represents total multi-pass small signal gain factor for the amplifier. The Equations (9) and (10) can be presented in diminished form: $g_{a f}=\hat{g}_{a}\left(g_{a i}, \varepsilon_{s}, \tau\right)$ and $\varepsilon_{f}=\hat{\varepsilon}\left(g_{a i}, \varepsilon_{s}, \tau\right)$, simplifying further operation with these formulas. Now, as the basic terms have been introduced, we can summarize a rule for subscript notations. The pump and amplification operation phases are designated with $p$ and $a$ subscripts respectively; the initial and final states are notated with $i$ and $f$ subscripts correspondingly.

\subsection{Coupling of successive cycles. Discrete-time dynamical system approach}

Evaluation of the output energy is a trivial task for low repetition rates, i.e. when the pump phase duration significantly exceeds the inversion relaxation time, $T>>T_{1}$. The gain in this case reaches saturation before the amplification phase starts, that is the initial gain $g_{a i}$ tends to unity. Consequently, the output energy versus round trip number is strictly defined by Eq. (10) alone. In general case, and particularly at high pulse repetition rate the initial 
conditions for the current operation stage depend on the previous system state. Hence, the initial gain for each cycle depends not only on operation parameters but also on the system pre-history. In order to determine the gain at the beginning of amplification we shall relate final and initial states of successive operation cycles.

Now we proceed from evaluation of the gain and pulse energy within single operation stages to a description of those stages as a whole by analyzing the temporal evolution of the boundary values. We assign initial and final gains and pulse energy as variables defining the system state. The term $g_{a i}(1)$ is introduced as the initial gain of the amplification phase for the first cycle of operation. This stage finishes with the final gain denoted as $g_{a f}(1)$. The subsequent pump phase of the current cycle obviously begins from the same gain value, $g_{p i}(1)=g_{a f}(1)$. Similarly, the gain evolution continuity should be taken into account for coupling of all operation cycles. There is a boundary relation $g_{a f}(k)=g_{p i}(k)$ within the cycle number $k$ and for subsequent cycles: $g_{p f}(k)=g_{a i}(k+1)$. The legend of the gain evolution in a discrete time scale can be presented as follows:

$$
\ldots g_{a i}(k) \rightarrow g_{a f}(k)=g_{p i}(k) \rightarrow g_{p f}(k)=g_{a i}(k+1) \rightarrow g_{a f}(k+1)=g_{p i}(k+1) \rightarrow g_{p f}(k+1) \ldots
$$

The corresponding time boundary points of neighboring operation stages can be described as $t_{a i}(k)=(k-1)\left(\tau T_{0} / G_{0}+T\right)$ and $t_{a f}(k)=k \tau T_{0} / G_{0}+(k-1) T$. Note that in an assumption of short amplification phase, the duration of complete cycle (dumping period) is approximately equal to the pump phase duration $T$. Hence the term $\left(T / T_{1}\right)^{-1}$ represents normalized pulse repetition rate of the regenerative amplifier (also called dumping frequency).

Analogous transition to the discrete time scale can be applied to energy evolution. However, unlike continuity of the gain evolution, the build-up of intra-cavity energy $\varepsilon_{s} \rightarrow \varepsilon_{f}(k)$ interrupts at the end of the amplification phase of each cycle at the moment of pulse dumping and then it begins again from the constant level which corresponds to the seed pulse energy $\varepsilon_{s}$. Hence the term $\varepsilon_{f}(k)$, determining the output energy, does not depend of its own pre-history. It is dependent on the gain and can be found from Eq. (10) for any operation cycle provided that the gain is known. Therefore, the gain becomes the only independent variable that needs to be analyzed.

We can consider the expressions obtained earlier $\left[\hat{g}_{p}\right.$ for the pump phase and $\hat{g}_{a}$ for the amplification phase explicitly represented by Eq. (6) and Eq. (9)] as the rules of the system state updating. These solutions of the rate equations serve as transformation rules that take the current state as input and update it by producing a new state. This new output state becomes the input for the next stage of operation. Then it is possible to combine amplification and pump phases within certain operation cycle and to form a joint gain transformation rule. We introduce $\hat{g}_{\Sigma}$ as the composition of functions $\hat{g}_{a}$ and $\hat{g}_{p}$ exhibiting the gain transformation rule for the complete cycle, $\hat{g}_{\Sigma}=\hat{g}_{p}\left(\hat{g}_{a}\right)$. Then using expressions of the inner functions we can present an explicit form of the function $\hat{g}_{\Sigma}$ :

$$
\hat{g}_{\Sigma}\left(g_{a i}\right)=1-\left[1-\frac{g_{a i}}{1+\frac{\varepsilon_{s}}{g_{a i}} \exp \left(g_{a i} \tau\right)}\right] \exp \left(-\frac{T}{T_{1}}\right)
$$

Thus, we have reduced the regenerative amplification to the evolution of single variable (system state, $g_{a i}$ ) in a discrete time scale; and also we have found a rule of this variable 
updating. The basic properties of this updating function fit to the mathematical definition of so called maps [functions whose domain (input) space and range (output) space are the same]. Then the regenerative amplification can be described by using the theory of onedimensional discrete-time dynamical systems (one-dimensional maps) (Alligood et al., 1996). The sequence of the system states $g_{a i}(1), g_{a i}(2), \ldots g_{a i}(k) \ldots$ is called an orbit in terms of this theory. The orbits can be calculated by using a recurrent formula determining the subsequent state of the system in terms of the present state: $g_{a i}(k+1)=\hat{g}_{\Sigma}\left[g_{a i}(k)\right]$.

It is obvious that in a regular single-energy regime the gain depletion during the amplification phase should be compensated by restoring the population inversion during the pump phase. In terms of states evolution, the initial gain of the amplification stage eventually should iterate, i.e. there is a certain gain value (designated as $g_{1}$ ) such that the subsequent gains stabilize upon reaching that value, $g_{a i}(k+1)=g_{a i}(k)=g_{1}$. Consequently, the system eigenstate satisfying the condition $g_{1}=\hat{g}_{\Sigma}\left(g_{1}\right)$ should exist. The solution of this equation is known as a fixed point in the discrete-time dynamical system theory. Being exactly equal to the fixed point, the system state reproduces itself after each cycle that leads to operating in a regular manner. However, requirement of technical feasibility of such a regime establishes a more strict condition to be fulfilled. The system should return to the fixed point after some perturbation has occurred, in other terms, more common for theory of dynamical systems, the fixed point should be attracting. Thus, study of regenerative amplification is reduced to the analysis of conditions of the fixed point existence and its stability characterization.

Since the equation determining the fixed points is transcendental we start analysis of the system state evolution with the graphical illustration. For more intuitive presentation, the fixed point existence condition [ $g_{1}=\hat{g}_{\Sigma}\left(g_{1}\right)$ with the explicit form of $\hat{g}_{\Sigma}$ given by Eq. (12) ] is rearranged into the following form:

$$
1-\left(1-g_{1}\right) \exp \left(\frac{T}{T_{1}}\right)=\frac{g_{1}}{1+\frac{\varepsilon_{s}}{g_{1}} \exp \left(g_{1} \tau\right)} .
$$

The right-hand part of this expression represents the gain transformation function during amplification, $\hat{g}_{a}$ [see Eq. (9)]. The left-hand part may be regarded as an inverse function of the gain recovery during the pump phase [transformed Eq. (6) gives $\left.g_{p i}=\hat{g}_{p}^{-1}\left(g_{p f}\right)\right]$. Since gain continuity implies equality of the boundary states $\left(g_{a i}=g_{p f}\right.$ and $\left.g_{a f}=g_{p i}\right)$ we can combine both curves $g_{a f}$ versus $g_{a i}$ and $g_{p i}$ versus $g_{p f}$ on a common diagram (Fig. 2). A space of system states, defined by this means, can give an intuitively simple but strict and fruitful picture of the system state evolution. The intersection of those curves, having clear physical meaning, gives solution of Eq. (13), i.e. it determines the fixed point of the system. It is important that the intersection of these curves always exists and it is always single for any set of control parameters. Really, the amplification stage curve is single-peaked, it begins from zero and always lies under the state space diagonal $\left(g_{a f}=g_{a i}\right)$. The letter is natural because during the amplification stage (provided, as we assumed, negligible pumping contribution) the population inversion is depleting by transforming to the intracavity pulse energy and respectively the gain can only decrease, $g_{a f}<g_{a i}$. The pump stage curve is a straight line whose slope depends on the repetition rate. This curve begins in the right upper corner of 
the state space $[(1,1)$ point $]$ and also always locates under the state space diagonal. Note, the state space, due to proper normalizing, has dimensions of $(0-1) \times(0-1)$. Thus, these curves cannot help intersecting and they intersect only once. Moreover, since the basic properties of the curves are universal, a fixed point existence and uniqueness is not only the result of mathematical speculations obtained under certain approximations but also the consequence of inherent physical properties of regenerative amplification.

One of two necessary requirements for existence of a stable single-energy regime, namely the fixed point uniqueness, is fulfilled and then the main concern is the fixed point stability study. Figures 2(a) and 2(b) represent diagrams of system state evolution for two typical cases. Figure 2(a) presents the orbit converging into an attracting fixed point. Such a convergence means that the regenerative amplifier eventually (after sufficient number of reiterations, when initial value of the orbit is "forgotten") starts producing regular pulsing. It is intuitively seen that the behavior of the resulting orbit (convergent or non-convergent) depends on the slope of the "amplification" curve in the fixed point with respect to the slope of the "pump" curve. Strictly speaking, the fixed point becomes attracting if the derivative of $\hat{g}_{\Sigma}$ function in this point satisfies the requirement $\left|\hat{g}_{\Sigma}^{\prime}\left(g_{1}\right)\right|<1$ (Alligood et al., 1996). The condition $\left|\hat{g}_{\Sigma}^{\prime}\left(g_{1}\right)\right|=1$ represents the transition point between stable and unstable operation. In case of $\left|\hat{g}_{\Sigma}^{\prime}\left(g_{1}\right)\right|>1$ the fixed point is repelling, and consequently stable operation becomes unfeasible.
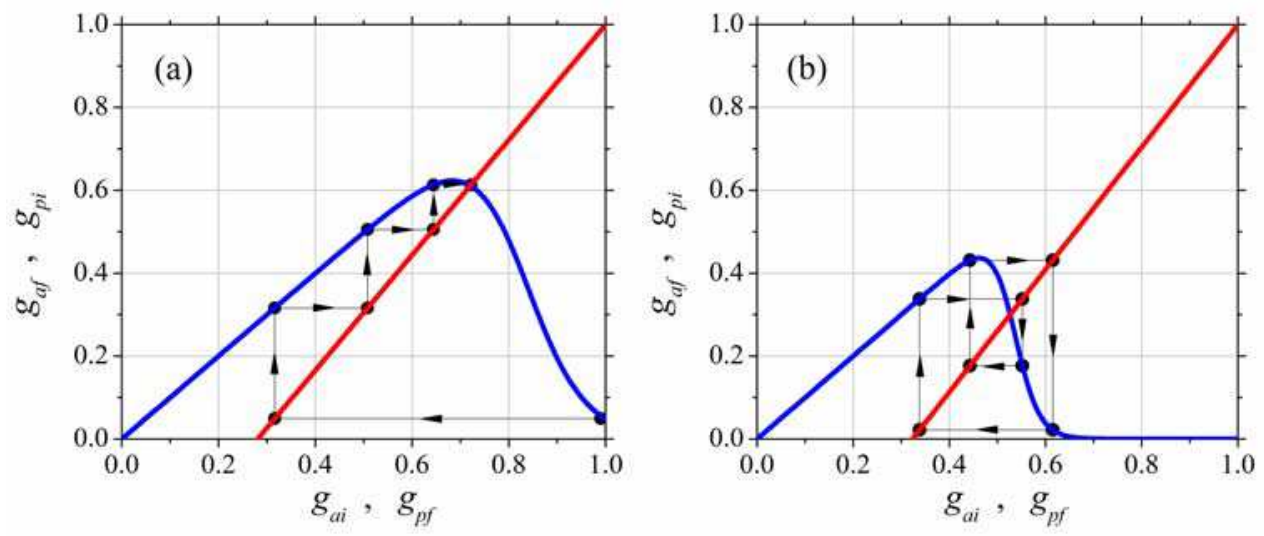

Fig. 2. Graphical presentation of the orbits in state space. The fixed point is the intersection of the "amplification" and "pump" (blue and red) curves. Transition to the stable (attracting) fixed point (a) at $\varepsilon_{s}=3 \times 10^{-7} ; \tau=18.0 ; T_{1} / T=3.0$. Period-4T orbit (b) at $\varepsilon_{s}=10^{-10}$; $\tau=42.0 ; T_{1} / T=2.56$.

It becomes apparent that in the latter case the system is unable to reproduce its own state after one cycle of operation. However, such a iteration may occur after two or several cycles. The corresponding set of system states is called periodic orbit. An example of periodic orbit is depicted in Fig. 2(b). The condition for existence of the orbit with double period, $2 T$ can be written by introducing an appropriate composite function. Define $\hat{g}_{\Sigma}^{2}=\hat{g}_{\Sigma}\left(\hat{g}_{\Sigma}\right)$ to be the result of applying the map-function $\hat{g}_{\Sigma}$ to the system state two times. The system state $g_{2}$ ， 
such that $g_{2}=\hat{g}_{\Sigma}^{2}\left(g_{2}\right)$, is the fixed point analogue but suited for two successive operation cycles. Generally the orbit with the period of $m T$ exists if there is a system eigenstate $g_{m}$ satisfying the equation: $g_{m}=\hat{g}_{\Sigma}^{m}\left(g_{m}\right)$. Here the term $m$ is an integer number exhibiting a factor of output pulse repeatability for the corresponding multi-energy regime. If such a regime is realized, the system produces quasi-periodic sequence of the output pulses. The pulses of identical magnitude in this sequence appear each time in a multiplied period equaled to $m T$. In much the same way as the existence of a fixed point does not ensure stable operation, the existence of a periodic orbit does not in itself mean that the corresponding regime is realizable. Additional analysis of the orbit stability is required, that, similar to the fixed point case, reduces to evaluation of the corresponding map-function derivative. The orbit $g_{a i}(k)$ of period- $m$ is stable provided that $\left|\left(\hat{g}_{\Sigma}^{m}\right)^{\prime}\left(g_{m}\right)\right|<1$. Computation of the derivative for this composite function is feasible since its value eventually (at $k \rightarrow \infty$ ) tends to the product of its inner function derivatives at points along the orbit: $\left(\hat{g}_{\Sigma}^{m}\right)^{\prime}\left(g_{m}\right)=\hat{g}_{\Sigma}^{\prime}\left[g_{a i}(1)\right] \hat{g}_{\Sigma}^{\prime}\left[g_{a i}(2)\right] \cdots \hat{g}_{\Sigma}^{\prime}\left[g_{a i}(k)\right]$. If the absolute value of the product of the derivatives is larger than one, then periodicity of the orbit becomes unfeasible meaning that the system exhibits chaotic behavior. This is the Lyapunov number criterion of deterministic chaos (Alligood et al., 1996).

We can remind here that the map-function $\hat{g}_{\Sigma}$ is itself a function of system parameters $\left(\tau, \varepsilon_{s}, T / T_{1}\right)$. As one of the governing parameters is varied the corresponding fixed point passes through different states of stability. A pass through the position $\left|\left(\hat{g}_{\Sigma}^{m}\right)^{\prime}\left[g_{a i}(k)\right]\right|=1$ causes qualitative change of the system operation. Such transitions (e.g. transition from stable to unstable regime at $m=1$ ) is usually called a bifurcation. A set of control parameters (operation point) at which the bifurcation occurs is referred to as the bifurcation point. Correspondingly the diagram of the output parameter versus one of the control parameters for the system exhibiting bifurcations is often called a bifurcation diagram. Among many possible types of bifurcations, known for dynamical systems, we have met here the bifurcation of period doubling. This relatively simple type of dynamic behavior is one of the consequences of the fixed point uniqueness. This attribute gives also primary unambiguity of the system behavior. The dynamics of regenerative amplification and output characteristics of the system are determined by the set of control parameters alone in contrast to e.g. bi-stability effects where initial value of the orbit, $g_{a i}(1)$ may also influence the operation. One can imagine the latter as qualitative change of system behavior caused by a way of switching it on (e.g. in practice either one has the pumping source enabled first and then seeding or other way around). The unambiguous relation between control parameters and operation regimes is quite an important property of regenerative amplifiers and our further analysis always implies this property without necessarily mentioning it.

\subsection{Diagrams of dynamic regimes in the parameter space}

Simplifying assumptions and non-dimensional effective parameters, introduced for the basic rate equation model, reduce the number of independent control parameters of the system to the set of three. These are the normalized repetition rate, $T_{1} / T$, amplification phase duration expressed in terms of the effective round trip number $\tau$ and normalized seed energy $\varepsilon_{s}$. Analysis of stability for orbits of the initial gain of amplification phase $\left[g_{a i}(k)\right]$ at each given control parameter provides thorough picture of regenerative amplifier behavior. 
The orbits were calculated by iterating of Eq. (12) in the range of control parameters wide enough to comprehend all the relevant dynamics features: $0.2<\left(T_{1} / T\right)<20 ; 10<\tau<110$; $10^{-11}<\varepsilon_{s}<10^{-3}$. We used as much as 3000 iterations, the sufficient number to be confident that the results are independent of the system state at the beginning of iterating. The orbits were analyzed in two stages. At first, the minimal number of cycles between repeating system states was revealed for each orbit in parameter space. It was performed by direct comparison of the system state sequences with themselves but shifted by a certain cycle number, $g_{a i}(k)$ versus $g_{a i}(k+m)$. In that way the periodic orbits up to $m=32$, including regular ones $(m=1)$, were identified. Then the Lyapunov number criterion was applied to the residual unidentified orbits. They were separated into two fundamentally different bunches: chaotic and eventually periodic.
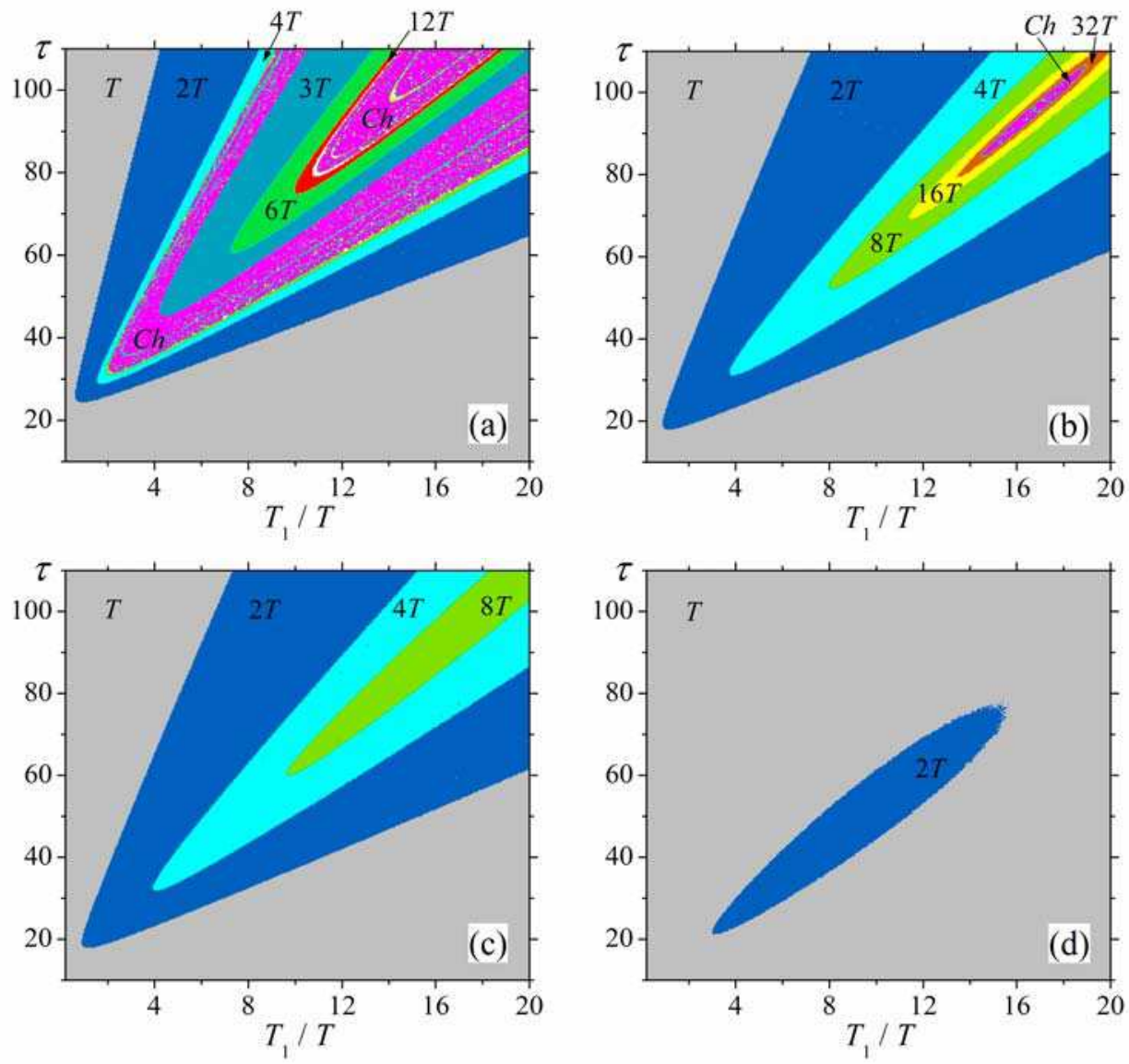

Fig. 3. Diagrams of amplification dynamics in parameter space for different seed energies: $\varepsilon_{s}=10^{-10}(\mathrm{a}) ; \varepsilon_{\mathrm{s}}=2.5 \times 10^{-7}(\mathrm{~b}) ; \varepsilon_{\mathrm{s}}=3 \times 10^{-7}(\mathrm{c}) ; \varepsilon_{\mathrm{s}}=1.3 \times 10^{-4}(\mathrm{~d})$.

Thus, the following dynamic regimes were distinguished in three-dimensional space of control parameters in accordance with the orbits properties: (1) The orbits evolving into 
stable fixed points $(m=1)$ corresponding to the regular system behavior (single pulse energy output, i.e. 1T-regime). (2) Periodic orbits corresponding to multi-energy regimes with repeatability coefficients in the range of $2 \leq m \leq 32$. (3) Eventually periodic orbits having larger repeatability factor $(m>32)$, for which the $m$-number itself is not identified. (4) Regime of deterministic chaos in accordance with the Lyapunov number criterion.

The regions of different dynamics are mapped in space of the repetition rate - round trip number (Fig. 3). The major part of the parameter space is occupied by the regions corresponding to the following regimes: single-energy (1T); quasi-periodic with fundamental period of two $(2 T, 4 T, 8 T, 16 T$, and 32T); quasi-periodic with fundamental period of three $(3 T, 6 T$, and $12 T)$; and chaotic behavior. These domains are marked with different colors, whereas the rest of the space containing the remaining zones of eventually periodic orbits is left white. The boundaries between adjacent colors (i.e. between different regimes) represent manifolds of bifurcation points in parameter space.

As it is seen, the dynamics turned out multifarious. Chaotic regime ordinarily comes out from the chain of successive period doubling bifurcations: T-2T-4T-8T-16T-32T... The chaotic zone itself has fine structure. Quasi-periodic "windows" with various periods are disseminated in it. The dynamics of regenerative amplification strongly depends on the seed value. The pattern is complex for low seed level $\left(\varepsilon_{s}<10^{-9}\right)$, the parameter space contains more than one clearly distinguishable chaotic regions [Fig.3(a)]. Quasi-periodic regimes with fundamental period of three are observed between zones of chaotic dynamics. The higher the seed energy, the simpler the instability pattern becomes. Initially, chaotic domain shrinks to ellipse [Fig. 3(b)] and disappears from the parameter space. Furthermore, period doubling bifurcations with fundamental period of two only remain for $\varepsilon_{S}>2.52 \times 10^{-7}$. Then the maximum order of bifurcations decreases [Figs. 3(c) and 3(d)] and finally, at $\varepsilon_{s}>1.9 \times 10^{-4}$ the system becomes stable in the whole range of control parameters.

\subsection{Seed pulse energy effect}

The obtained results, demonstrating dependence of the operation on the seed pulse energy, are in essence not quite trivial. This phenomenon is in controversy with intuitive comprehension of regenerative amplification. The following speculations seem to demonstrate the negligible extent of the seed influence or at least to evidence much simpler looking relations. Imagine, initially low seed pulse energy $\varepsilon_{s 1}$ after certain number of round trips $(\Delta \tau)$ is amplified to energy $\varepsilon_{s 2}$ of several orders of magnitude larger but still much less than energy stored in the gain media, $g_{a i}>>\varepsilon_{s 2}>>\varepsilon_{s 1}$. Then further amplification should give the same output as if the amplification has initially started with seed energy $\varepsilon_{s 2}$ because the previous stage $\left(\varepsilon_{s 1} \rightarrow \varepsilon_{s 2}\right)$ virtually has not changed the stored energy and, as a consequence, the system gain. This logic leads to an inference that a lack of seed energy can be compensated with additional round trips. Consequently, the operation diagrams have to look identical but shifted in the coordinate of round trip number for different seed values. Accurate computations give absolutely different results, Fig. 3.

Let us consider in details some subtleties which lead to this difference. In time-domain the amplification process looks exactly as simple logic predicts, that can be confirmed with straightforward calculations. Equation 10 gives equal but shifted in time intracavity energy evolutions, $\varepsilon\left(g_{a i}, \varepsilon_{s 1}, t^{\sim}\right)=\varepsilon\left(g_{a i}, \varepsilon_{s 2}, t^{\sim}-\Delta \tau\right)$ and this shift, $\Delta \tau$ can be determined as: 
$g_{a i} \Delta \tau=\ln \frac{g_{a i}}{\varepsilon_{s 1}}-\ln \frac{g_{a i}}{\varepsilon_{s 2}}$. This logic is accurate when we are considering amplification phase as "isolated" with given initial gain; this is absolutely true for low repetition rates.

Previously it was commonly accepted that regenerative amplification virtually is independent of the seed energy because only low repetition rates were under consideration. In reality, even for low repetition rate systems the seed energy value should not be too low. However, the reason for that is rather different of what we are describing. Simply, competing parasitic processes of amplification of spontaneous emission always exists in regenerative amplifiers. Thus, the seed energy should be well above the spontaneous emission level in order to get the amplified seed at the output instead of amplified spontaneous emission. However, at low repetition rates the sufficient pulse seed energy is extremely small, e.g. down to $10^{-15} \mathrm{~J}$ in accordance to the experiments presented in the classical paper (Murray \& Lowdermilk, 1980). Although, some exceptions may take place; they involve special applications which require very clean, high contrast optical pulse, e.g. parametric chirped pulse amplification (Ross et al., 2007).
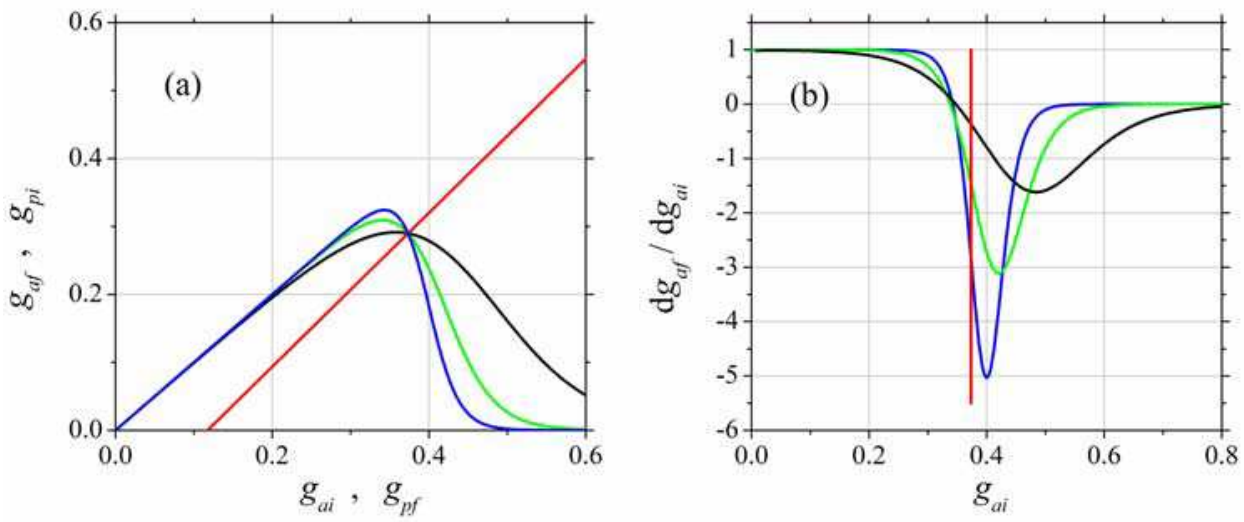

Fig. 4. Typical state space diagrams of regimes having equal fixed points at different seed energies (a) and derivatives of the "amplification" curves (b).

It becomes apparent that at high repetition rates the initial gain depends on preceding operation cycles and can be determined indeed by taking them into account. This procedure is in essence nothing else than the fixed point determination. Let us return to geometric presentation of fixed points in state space. Figure 4(a) represents diagrams of the final gain against initial gain for three seed energy values. Corresponding numbers of round trips have been selected so that the fixed points at certain repetition rate are identical. All the curves intersect in a single point which corresponds to the fixed point for the repetition rate $T_{1} / T=8.0$ This means that decrease in seed energy is compensated by increasing of round trips but only in a sense of equal position of the fixed points. However the shapes of curves $g_{a f}$ versus $g_{a i}$ are different and their derivatives in the intersection point are dependent on the seed energy [Fig. 4(b)]. Such derivatives indeed determine the regenerative amplification stability as we have described in section 2.4 by referring to the theory of discrete-time dynamical systems.

The identity of the fixed points can be realized by compensation of the seed energy difference by appropriate selection of the round trip number. Output energies 
corresponding to those fixed points are equal too. However the peculiarity of operation at high repetition rates is such that dynamical system behavior is absolutely different. Thus at high repetition rates the seed pulse energy becomes one of those critical parameters which determine the operation regime of the regenerative amplifier. The specific operation points, which have been analyzed here, can be also found in the diagrams of dynamic regimes (Fig. 3). With regard to stability, they were classified as stable, $2 T$-periodic and chaotic for seed values of $1.3 \times 10^{-4}, 2.5 \times 10^{-7}$ and $10^{-10}$ respectively. The dynamic regimes which are in general possible to obtain (by changing the round trip number) within a certain range of seed values are summarized in Table 1 .

\begin{tabular}{|l|l|l|}
\hline \multirow{2}{*}{ Existing regimes } & \multicolumn{2}{|c|}{ Seed value range } \\
\cline { 2 - 3 } & \multicolumn{1}{|c|}{$g_{t}=0$} & \multicolumn{1}{c|}{$g_{t}=0.028$} \\
\hline Chaos and “all" periods & $<2.52 \times 10^{-7}$ & $<1.4 \times 10^{-5}$ \\
\hline$T, 2 T, 4 T, 8 T, 16 T, 32 T \ldots$ & $2.52 \times 10^{-7}-2.56 \times 10^{-7}$ & $1.4 \times 10^{-5}-1.5 \times 10^{-5}$ \\
\hline$T, 2 T, 4 T, 8 T, 16 T$ & $2.56 \times 10^{-7}-2.72 \times 10^{-7}$ & $1.5 \times 10^{-5}-1.74 \times 10^{-5}$ \\
\hline$T, 2 T, 4 T, 8 T$ & $2.72 \times 10^{-7}-3.56 \times 10^{-7}$ & $1.74 \times 10^{-5}-2.5 \times 10^{-5}$ \\
\hline$T, 2 T, 4 T$ & $3.56 \times 10^{-7}-1.39 \times 10^{-6}$ & $2.5 \times 10^{-5}-4.1 \times 10^{-5}$ \\
\hline$T, 2 T$ & $1.39 \times 10^{-6}-1.90 \times 10^{-4}$ & $4.1 \times 10^{-5}-3.5 \times 10^{-3}$ \\
\hline$T$ (stable) & $>1.90 \times 10^{-4}$ & $>3.5 \times 10^{-3}$ \\
\hline
\end{tabular}

Table 1. Possible regimes versus seed pulse energy range.

\subsection{Influence of parasitic intracavity losses on dynamic pattern}

The approximation of negligible losses is a good way to present the main ideas for application of the discrete-time dynamics method for regenerative amplification and to understand the dynamic patterns most relevant at high repetition rates. However, this approximation has limited application in practice. The output pulse energy grows in the lossless system monotonically together with amplification phase duration and reaches saturation at the level of $\varepsilon_{\max }=1-\exp \left(-T / T_{1}\right)$ that corresponds to full conversion of stored energy (population inversion) to the output pulse energy. Consequently the number of round trips can be increased, without detriment to output energy, to the values high enough for operation behind the bifurcation zone that in turn assures stable operation. Actually, the system is always (i.e. irrespective of losses) stable provided that the population inversion is well depleted during the amplification phase. In this case the initial gain tends to the constant, determined only by the repetition rate, $\left[g_{a f} \rightarrow 0 \Rightarrow g_{p i} \rightarrow 0\right.$; then from Eq. 6 follows that $\left.g_{a i}=g_{p f} \rightarrow 1-\exp \left(-T / T_{1}\right)\right]$. Consequently, the interdependence of operation cycles vanishes that results in eliminating of immediate cause of unstable behavior. In reality, parasitic losses prevent utilization of this property since because of losses the mode of complete gain depletion becomes inefficient.

Well known efficiency criterion, to dump optical pulse off the resonator at the moment when the current gain has dropped down to the threshold gain $\left(g_{a f}=g_{t}\right)$, is not applicable to repetitive operation as relating to only "isolated" operation cycles. Power efficiency enhancement takes place at high repetition rates when stored energy is left partially underdepleted $\left(g_{a f}>>g_{t}\right)$ forming a substantial gain background after several operation cycles. The proportion of gain to losses, which eventually determines extraction efficiency of the stored energy, can be well improved by this means. However incomplete depletion is an origin of 
operation cycles interdependence therefore in presence of losses the system efficiency in some sense collides with the system stability.

Parasitic losses in laser systems are given by optical components imperfection and diffraction losses of the optical resonator. The latter are objects of resonator geometry optimization. In case of solid-state lasers pumped longitudinally (virtual absence of hard apertures) high order optical aberrations (spherical e.g.) may become the main origin of diffraction losses, especially at high pumping intensities (Liu C. et al. 2008). Among optical components, the electro-optic switch is usually the most critical part; contributions of the Pockels cell and the polarizer to the loss factor surpass the remaining components (Müller et al., 2003). Practically, the level of total parasitic losses can vary in quite a wide range, but the typical value should not exceed a few percent per roundtrip for high-quality, well optimized systems. Here we should remark that the losses, inherent for quasi three-level gain media and related to partially populated ground state, are not dissipative and they do not belong to the parasitic losses which we are considering.

At the account of intracavity losses, general, qualitative pattern of amplification dynamics (fixed points uniqueness, variety of orbits for the repulsive fixed points, significance of the seed pulse energy) remains the same, but naturally the quantitative difference factors in. The intracavity losses of regenerative amplifier not only reduce efficiency (that is natural for lasers), but also substantially interfere in total system stability.
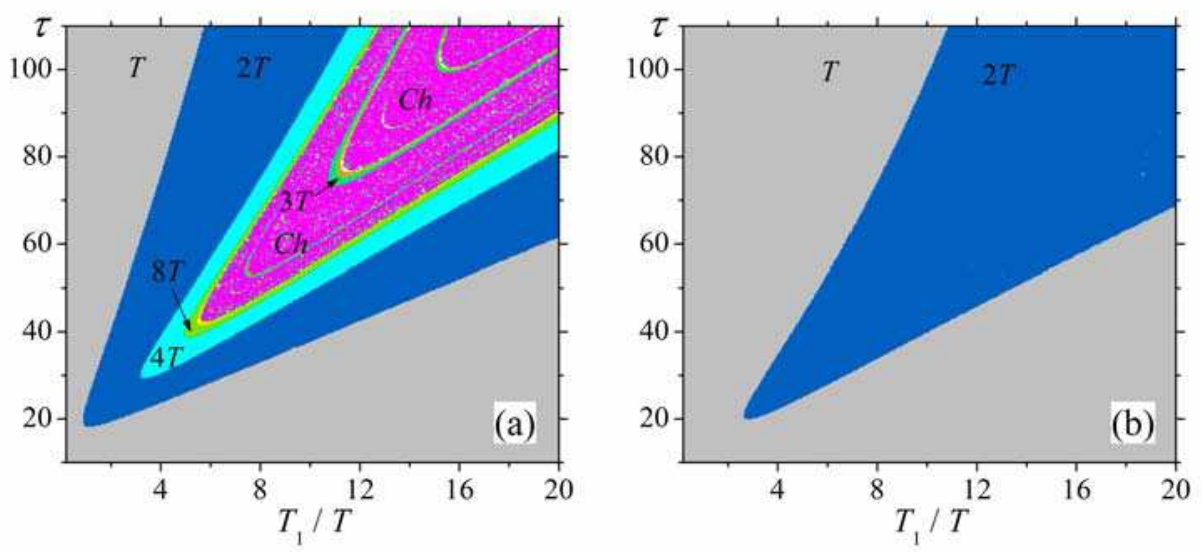

Fig. 5. Diagrams of amplification dynamics for threshold gain $g_{t}=0.028$ and seed pulse energies $\varepsilon_{s}=3 \times 10^{-7}(\mathrm{a}) ; \varepsilon_{\mathrm{s}}=1.90 \times 10^{-4}(\mathrm{~b})$.

The diagrams of regenerative amplification dynamics in parameter space (round trips repetition rate) for intracavity losses corresponding to the threshold gain $g_{t}=0.028$ are presented in Fig. 5. Fixed point calculation and their stability evaluation were performed analogously as described in sections 2.4 and 2.5. The only difference is function $\hat{g}_{a}$, relating final and initial gains [solutions of Eqs. (4) and (7)], was calculated numerically since analytical solution is unknown in case of nonzero losses. The diagram presented in Fig 5(a) can be compared with that given for the same seed pulse energy $\varepsilon_{s}=3 \times 10^{-7}$ but for zero losses [Fig 4(c)]. The influence of losses results in a more complicated dynamic pattern; the zone of chaotic dynamics evolves and the high order bifurcations shift closer to the tip of the 
instability zone (towards lower repetition rate and lower round trip number). The second diagram [Fig 5(b)] was calculated for the seed energy $\varepsilon_{s}=1.90 \times 10^{-7}$, which at zero losses provided stable operation in the whole range of control parameters. Now a period doubling zone $(2 T)$ has occupied a certain part of the parameter space and noticeably narrowed the range of stable operation.

A more cumulative picture of dynamical regimes is presented in Table 1. One can see some general change for the worse for system stability with respect to the zero-loss case. The decrease in stability caused by losses is not an obvious phenomenon (why not increase?). The reason for that bears similarity to the seed energy effect. The losses decrease pulse energy addition per round trip that can be compensated by increasing of round trip number, but only in a sense of fixed point identity. The derivative magnitude of the gain transformation function, $\left|\hat{g}_{\Sigma}^{\prime}\left(g_{1}\right)\right|$, in this point has changed so that system stability becomes worse as the losses are increasing. This phenomenon is not obvious but the conclusion is straightforward - the parasitic losses should be minimized as much as technically possible not only for efficiency but also for better stability.

\subsection{System performance representation in parameter space}

For understanding regenerative amplifier performance, not only dynamical regimes but also output pulse energy should be represented in relation to the parameters governing the system. In previous sections we have described in detail the method of fixed points and related orbits determination. Actually, during calculation of the initial gain orbit, $g_{a i}(k)$, the orbit of output energy $\varepsilon_{f}(k)$ comes out automatically as the second solution of coupled Eqs. (4) and (7), $\varepsilon_{f}(k)=\hat{\varepsilon}\left[g_{a i}(k), \varepsilon_{s}, \tau, g_{t}\right]$. If governing parameters are such that operation is unstable then the initial gain varies from cycle to cycle by specific means and consequently the output energy becomes a multi-valued function of governing parameters. Representation of such a function on $2 D$ diagram is unfeasible, it looks like a mess. Therefore we present typical dependencies of output energy versus round trip number (bifurcation diagrams) at several repetition rates for the fixed loss factor (corresponding to the threshold gain $g_{t}=0.028$ ) and seed energy $\varepsilon_{s}=7.7 \times 10^{-7}$ (Fig. 6). The selected seed pulse energy belongs to the same range that typical for functionally important class of seed lasers operating in CW mode-locking regime with moderate average power (around hundreds milliwatt). The repetition rates are chosen according to the diagram of dynamic regimes [Fig. 6(a)] so that typical bifurcation diagrams up to 16T-regime are demonstrated.

It is seen that the output energy variation in the presence of period doubling is so high that it virtually leaves no opportunity to use such a regime in practical applications. For example at repetition rate $T_{1} / T=4.6$ and in 30-60 round trips range the output energy alternates between high and low value so badly that the output pulse train looks almost as at twice less repetition rate [Fig. 6(c)]. It is even more pity that such bad stability often appears in regimes which potentially capable of providing high output energies.

The maximum capability of the system can be determined by calculating the output energy exactly in the fixed point: $g_{a i}=g_{1} \Rightarrow \varepsilon_{1}=\hat{\varepsilon}\left(g_{1}, \varepsilon_{s}, \tau, g_{t}\right)$. This energy is always a single-valued function of the governing parameters regardless of whether the fixed point is attractive or repulsive. In case of a repulsive fixed point (i.e. unstable operation), the corresponding "fixed point" output energy becomes an artificial parameter but it can serve as a convenient reference for evaluating the power efficiency reduction caused by instability effects. 


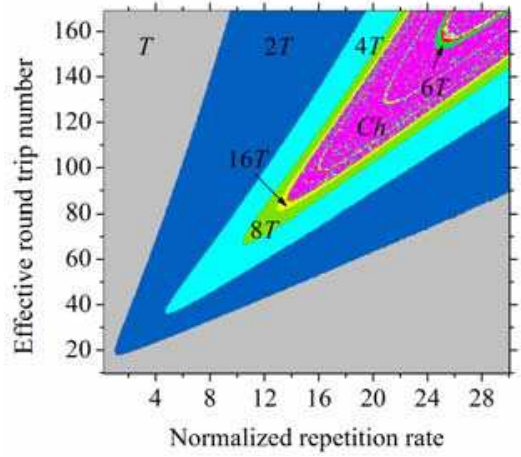

(a)
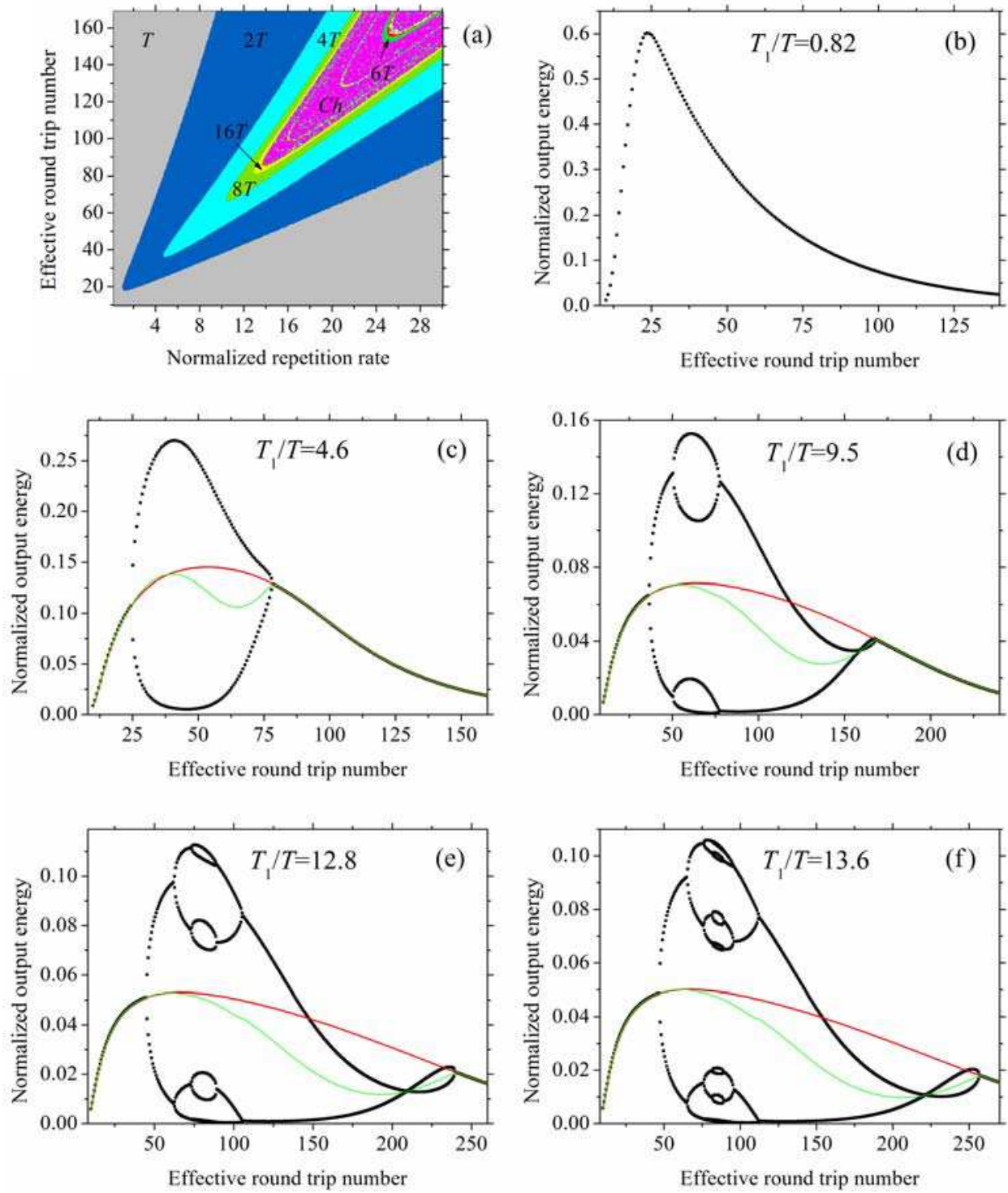

Fig. 6. Diagram of dynamic regimes (a) and corresponding bifurcation diagrams for selected repetition rates $(\mathrm{b}-\mathrm{f})$ at $\varepsilon_{s}=7.7 \times 10^{-7}$ and $g_{t}=0.028$. Pulse energy, averaged pulse energy and "fixed point" energy correspond to black, green and red lines respectively.

We also determined real (accounting multi-energy nature) output energy averaged over large number of operation cycles $\left\langle\varepsilon_{f}(k)\right\rangle$ (Fig. 6). Interestingly, the real averaged energy is considerably lower than the reference "fixed point" energy in regimes exhibiting pronounced period doubling. The question is "what can the origin of this energy defect be?" 
One can suppose that in a multi-energy regime a relatively larger part of the resonator energy circulating is redistributed to the channel of parasitic losses. Some evidence for this explanation is that the same curves calculated in case of zero losses coincide in spite of bifurcations. The only alternate reason is stored energy depletion through spontaneous emission during the pump phase. However, the latter effect becomes dominant when the population inversion is on average high (i.e. at low round trips), that is not the case now.
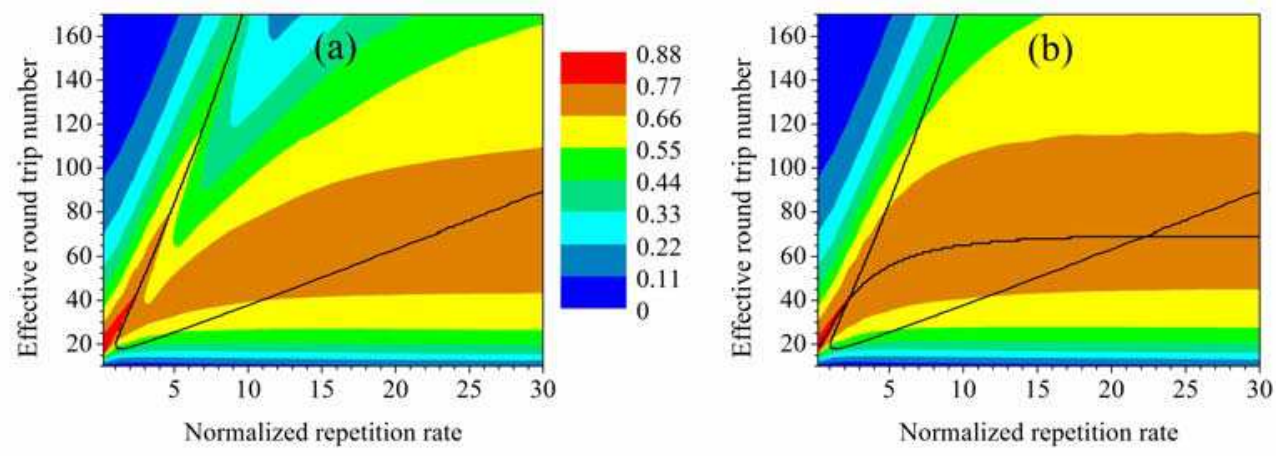

Fig. 7. Efficiency of stored energy extraction with respect to the separatrix (a); the reference ("fixed point") efficiency with respect to the separatrix and $\tau_{\max }$ curve (b); both diagrams are for $\varepsilon_{s}=7.7 \times 10^{-7}$ and $g_{t}=0.028$.

Single-valued functions characterizing output energy allow presentation of an informative picture of system performance in $2 D$ diagrams. The extraction efficiency of stored population inversion may serve as a more convenient parameter for this purpose. The extraction efficiency can be defined as averaged output energy divided by maximum stored energy available at a given repetition rate, $\left\langle\varepsilon_{f}(k)\right\rangle / \varepsilon_{\max }$, where $\varepsilon_{\max }=1-\exp \left(-T_{1} / T\right)$. Then the value calculated in the fixed point $\left(\varepsilon_{1} / \varepsilon_{\max }\right)$ can be regarded as the maximum attainable efficiency at a given round trip number. Typical diagrams of these parameters in the space of repetition rate versus round trip number are presented in Fig. 7. Additional normalization to $\varepsilon_{\max }$ introduced for already normalized terms is not meshing. Quite the contrary, it simplifies the practical use of theoretical data. The efficiency is the ratio which has the same value for real "dimensional" and normalized parameters.

Apparently, stable single-energy operation is the only suitable regime for routine use of regenerative amplifiers. For completeness sake we can note that one may successfully use certain unstable regimes for specific applications provided that there is comprehensive understanding of the essence of period doubling (Metzger et al., 2009). However this is rather the exception than the common rule. Thus, we can omit a detailed picture of dynamical regimes in order to move towards more pragmatic considerations. It is sufficient to leave only one curve in the parameter space defining the range of operating points in which operation is stable. This curve (further referred to as a separatrix) represents a manifold of the first order bifurcation points (1T-2T boundary) and actually separates zones of stable and unstable operation in the parameter space. Both real and "fixed point" energies are equal within a range of control parameters providing stable operation. Some drop of real efficiency with respect to that obtained in the assumption of stable operation is observed immediately below the upper branch of the separatrix, in the instability zone [Fig 7(a)]. The 
cross-sections of this feature are observed in Fig. 6 at several repetition rates and we have already concluded that this "valley" originates from enhanced influence of parasitic loses in the period doubling regime.

The distribution of the "fixed point" extraction efficiency $\left(\varepsilon_{1} / \varepsilon_{\max }\right)$ in the parameter space contains sufficient information to determine system performance when accompanied with the separatrix curve. This curve confines the space of stable operation that is the range where the "fixed point" data correspond to reality. For system optimization it is important to find operation points potentially providing maximum performance at each given repetition rate. The manifold of such points represents a curve of round trip number versus repetition rate which is mapping the peak value of the "fixed point" extraction efficiency ( $2 D$ distribution in parameter space) and further referred to as $\tau_{\max }$ curve [Fig $\left.7(\mathrm{~b})\right]$. The potential performance becomes real at repetition rates in which the $\tau_{\max }$ curve is outside of the instability region and when the round trip number is set equal to $\tau_{\max }$. If the $\tau_{\max }$ curve is inside the instability zone then the system capabilities are underexploited. The corresponding range of repetition rates can be called the "critical range". Within the critical range there are two possible positions of the operating point which may provide maximum output in the stable regime - the points along the lower and upper branch of the separatrix [see Fig 7(b)]. According to the diagram, at lower repetition rates in the critical range the upper branch has an advantage from an efficiency point of view. Operation at lesser round trip number (lower branch of the separatrix) becomes preferable at higher repetition rates. Thus optimization of the regenerative amplifier is actually reduced to selection of the round trip number which provides maximum output pulse energy and at the same time allows stable operation for the required repetition rate range(imposed by the system specifications). The corresponding round trip number is logical to call optimum $\left(\tau_{\text {opt }}\right)$. Obviously, $\tau_{\text {opt }}$ is equal to $\tau_{\max }$ outside the critical range.

Instructive inference of considered above regenerative amplification properties is that within critical repetition rates the round trip number takes on optimum value either near the lower or near the upper separatrix branch but always at the margin of unstable operation. In general, operation at the margin of stability incurs challenges for robust operation in real systems which undergo technical noises. Even slight changes to control parameters may result in system instability. Therefore reliably stable operation generally requires setting the operating parameters well away from the instability border, but this in turn leads to a reduction of the system performance.

There is an important parameter related to intracavity losses which may influence performance of regenerative amplifiers indirectly: the amount of intracavity energy dissipated during the amplification stage. Accumulated over round trips, the fraction of intracavity energy, dissipated through parasitic losses is subject to the specific operation regime. In particular, multiple passes of the already amplified optical pulse lead to substantial enhancement of energy dissipation. This, in turn, may give unacceptably high heating of intracavity components caused by the absorbed part of the dissipated power. One of the critical components in this respect can be the Pockels cell crystal. It may lose contrast under excessive heating possibly resulting in failure of regenerative amplifier operation. This effect is especially pronounced for systems intended for high power applications. The energy defect, arisen due to parasitic losses $\left(\varepsilon_{l}\right)$, can be determined as a product of intracavity energy, integrated over the amplification stage, and loss factor expressed by the threshold gain $g_{t}$. Using this definition and Eqs. (4) and (7) we get: 


$$
\varepsilon_{l}=g_{t} \int_{0}^{\tau} \varepsilon\left(t^{\sim}\right) d t^{\sim}=\int_{0}^{\tau} \varepsilon\left(t^{\sim}\right) g\left(t^{\sim}\right) d t^{\sim}-\varepsilon_{f}=g_{a i}-g_{a f}-\varepsilon_{f}
$$

where the integration variable $t^{\sim}$ is the current normalized time. The same result can be obtained from the energy conservation law since during short (as we have initially assumed) amplification stage there are only two energy consumption channels - useful signal and parasitic losses. In case of multi-energy regime the effective lost part of the energy can be found by averaging:

$$
\left\langle\varepsilon_{l}\right\rangle=\frac{1}{k} \sum_{k}\left(g_{a i}(k)-g_{a f}(k)-\varepsilon_{f}(k)\right)
$$

The diagram of dissipated energy (also normalized to maximum stored energy $\varepsilon_{m a x}$ ), for the set of governing parameters used before, is presented in Fig. 8(a). We can see that the large number of passes, typical for the upper separatrix branch, substantially contributes to this parameter. So the dissipated energy is about 7.5 times higher at the repetition rate when theoretical efficiencies for both branches are equal (at $T_{1} / T \approx 6.5$ ). This increase with respect to the lower branch often makes operation at high round trip number, well above $\tau_{\max }$ point inefficient, despite theoretical preference.
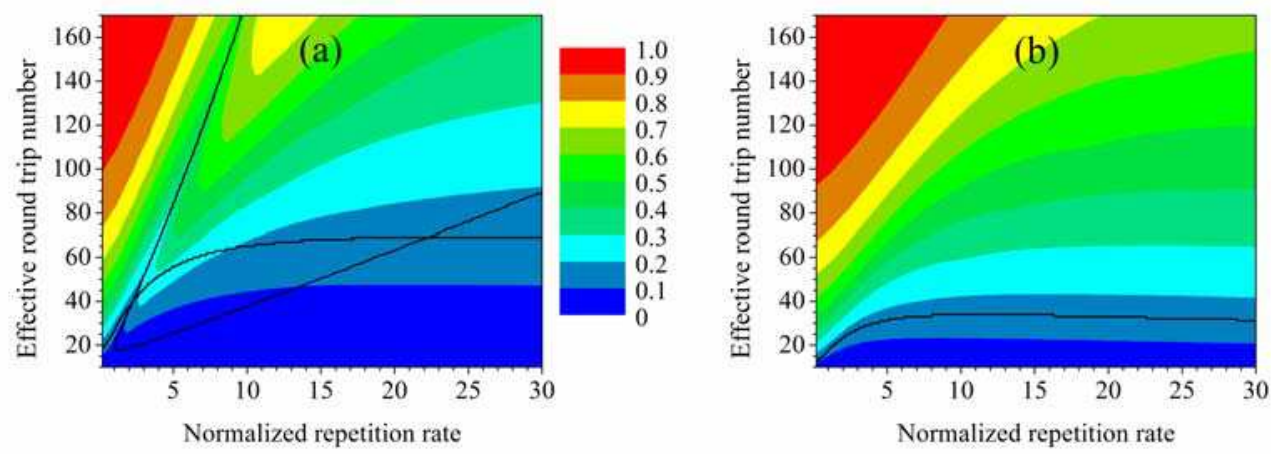

Fig. 8. Dissipated intracavity energy, separatrix and $\tau_{\max }$ curves for $g_{t}=0.028$ and $\varepsilon_{s}=7.7 \times 10^{-7}$ (a); dissipated energy, and corresponding $\tau_{\max }$ curve for the seed energy $\varepsilon_{s}=2.46 \times 10^{-4}(\mathrm{~b})$.

All the issues described in this section are regarded to one certain seed energy $\varepsilon_{s}=7.7 \times 10^{-7}$. The corresponding diagrams give a typical but single section of multidimensional space of control parameters. However, as we can already conclude from amplification dynamics data presented in sections 2.6 and 2.7, the critical repetition rate range depends on the seed pulse energy. Thus, the picture of regenerative amplification is still incomplete and it is time to proceed to consideration of system optimization taking into account influence of the seed pulse energy.

\subsection{Stability diagrams and pulse duration effects}

It is possible to present data which allow evaluation of regenerative amplification of different seed pulse energies by considering a single diagram. The necessary premises for doing that have been formulated in the previous sections. The condition where bifurcations 
are absent in the whole parameter space (Table 1) gives a general understanding of the seed energy influence; however this condition is too strong from a practical point of view. In order to thoroughly utilize power capabilities of the regenerative amplifier at a certain repetition rate, the round trip number should be set equaled to $\tau_{\max }$. Stable operation requires having the point $\tau_{\max }$ outside the instability zone (delimited by the separatrix curve on the parameter space). The inter-positioning of the separatrix and the $\tau_{\max }$ curve contains sufficient information to determine the critical repetition rate range for a certain seed energy and the possible optimum operation points $\left(\tau_{\text {opt }}\right)$ within this range (which lie, as we have known, along one of the separatrix branches). The diagrams consisting of $\tau_{\max }$ curves and separatrixes (further referred to as stability diagrams) for selected pulse seed energies are presented in Fig. 9.
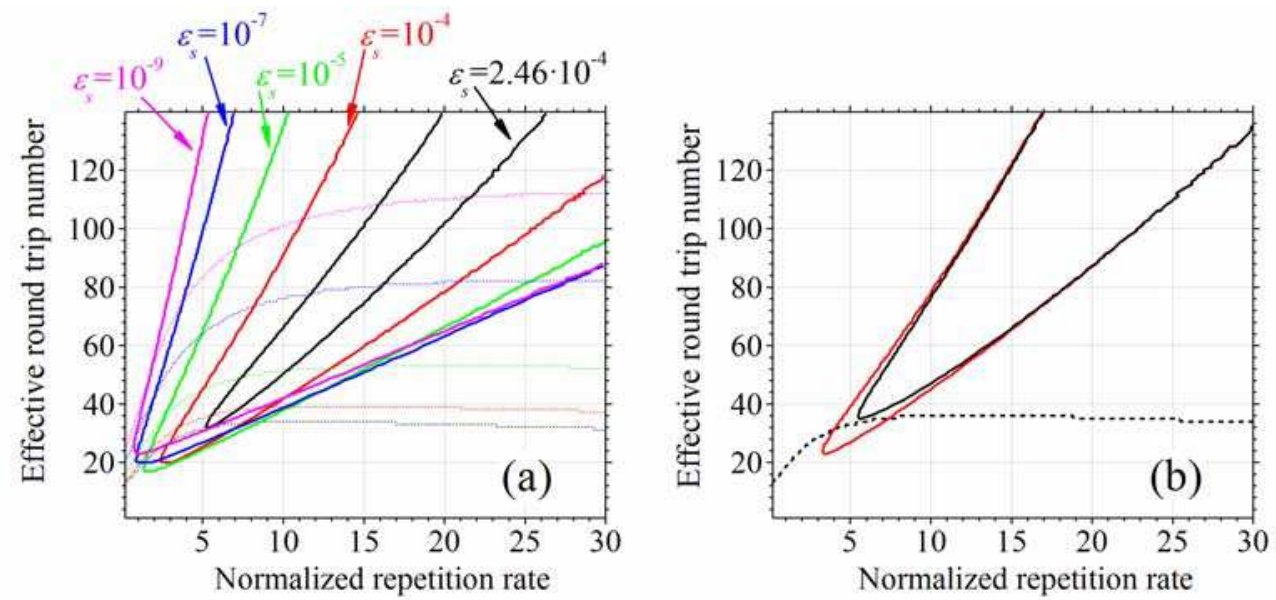

Fig. 9. Stability diagrams for the threshold gain $g_{t}=0.028$; the curves couples of the same color (the separatrix and $\tau_{\max }$ curve) correspond to certain seed energies (a). The separatrixes are shown for the short pulse (black solid line) and long pulse approximations (red solid line) and $\tau_{\max }$ curve (dash line) at $g_{t}=0.028$ and $\varepsilon_{s}=1.7 \times 10^{-4}(\mathrm{~b})$.

The approach of stability diagrams forms a more systematic concept of the system behavior and specifically allows estimation of the seed level which may enable one to avoid instability effects at the required pulse repetition rate. Also we can see that the critical range shrinks as the seed energy is increasing. The most critical repetition rate is $T / T_{1}=5.5$, the point of the "worst stability", requiring the highest seed energy for optimal operation. Finally one can determine the seed pulse energy at which $\tau_{\max }$ curve does not pass instability zone at all. For the specific parasitic losses that we consider $\left(g_{t}=0.028\right)$ this energy is equal to $2.46 \times 10^{-4}$. Consequently, a regenerative amplifier seeded with pulse energy higher than that value (further referred to as "ample") allows theoretically attainable average power and stable operation over the whole repetition rate range. Also we can mention here another advantage of the "ample" seed operation: As soon as the critical range has disappeared there is no need to operate at the upper separatrix branch and to suffer from large intracavity energy dissipation peculiar to this regime. The diagram of dissipated energy for the ample pulse seed energy, $\varepsilon_{s}=2.46 \times 10^{-4}$ is depicted in Fig. 8(b). 
The approach of stability diagrams is a straightforward way of regenerative amplifiers optimization. However in real systems there are specific effects which influence dynamic behavior and output parameters and they cannot be elaborated by using only approximation of simplified rate equations. Nevertheless it is possible to understand some important contributions staying basically within present approach.

The major application of regenerative amplifiers is amplification of short (even more common term is ultrashort) optical pulses. Now we shall consider the influence of pulse duration on regenerative amplification. The theory described above is based on rate equations formulated for idealized four-level system. One of the positions in the definition of that "ideality" is instant depopulation of the lower laser level (which is also called terminal level). In reality we can assume that the lower laser level is virtually unpopulated, only provided that the amplified optical pulse is much longer than the terminal-level lifetime (long pulse approximation). Otherwise, if the length of the optical pulse is much less than the terminal lifetime then the terminal level will remain populated resulting in "faster" decay of population inversion (indeed during amplification of a single pulse). Comprehensive evaluation of actual terminal-level lifetime provided by (Bibeau, et al., 1995) for different neodymium doped laser media gives actual values well exceeding $100 \mathrm{ps}$. So we can conclude that amplification of pulses shorter than 100ps virtually for all neodymium based systems is more appropriate to analyze within the short pulse approximation that is assuming negligible terminal-level depopulation during singe pulse amplification. This, in fact, constrains applicability of presently described approach and leaves beyond the scope functionally important ultrashort pulses. We can note in advance that net contribution of the terminal-lifetime effect to regenerative amplifier behavior is rather weak at high repetition rates. However it gives some quantitative refinements to the picture presented above.

In order to elaborate regenerative amplification in the short pulse approximation we need to re-calculate the fundamental relations of final and initial gains $g_{a f}=\hat{g}_{a}\left(g_{a i}\right)$. In this approximation the amplification of a single pulse was regarded as that in truly three-level gain media with initially empty ground state. After single pass amplification the ground state becomes partially populated, but by the second pass it is empty again and consequently the gain defect, which appeared due to "instant" three-levelness, is recovered. The latter is the case since we assume the terminal-level lifetime to be much shorter than the round trip time, $T_{0}$. Typical $T_{0}$ value is in the range between ten and a few tens of nanoseconds, so this is good approximation for most of neodymium media [actually except some fluoride crystals and glasses in which the neodymium terminal lifetime is of $10 \mathrm{~ns}$ order (Bibeau, et al., 1995)]. By this means and by using sequential procedure the basic relations $g_{a f}=\hat{g}_{a}\left(g_{a i}\right)$ and corresponding output energies were determined. The subsequent procedures (evaluation of the fixed points and their stability analysis) stay unchanged for the short pulse approximation.

We revealed that the influence of a terminal level appears as follows. There is noticeable deviation between the $\tau_{\max }$ curves at low repetition rates. However both "short pulse" and "long pulse" curves virtually coincide at the repetition rates $T_{1} / T>1.0$, indeed in the range that we are studying. The separatrix curves practically coincide at low seed energy levels, although filling of the instability zone differs from what have been seen earlier [e.g. in Fig 6(a)] towards not so wide variety of regimes. These peculiarities add little in practical essence. Therefore we have come to nothing more than qualitative description and statement of that fact. 
The only noticeable influence of the terminal lifetime was found at high seed energy, approaching to the ample level. The stability diagram, depicted in Fig.9 (b), shows what the difference is. In comparing two separatrixes we can point out that the tip of the "short pulse" separatrix is somewhat shifted towards higher round trip number and higher repetition rate. Such a shrink of the instability zone gives certain improvement of general system stability. The ample seed pulse energy determined for short pulses is almost 1.5 times less than that found within long pulse approximation $\left(\varepsilon_{\mathrm{s}}=1.7 \times 10^{-4}\right.$ against $\left.2.46 \times 10^{-4}\right)$. This improvement is rather unexpected result since terminal-level "bottleneck" in some respect hampers the ideal four-level amplification. Thus at least partially and shortly populated terminal level does not act as additional losses as one might imagine.

Another phenomenon, substantial at high intensities and therefore requiring intent attention while ultrashort optical pulses are amplifying, is the Kerr effect. This nonlinearity makes an impact on amplification process by intensity dependent refractive index change in volume intracavity components. The influence occurs in both spatial and spectral domains and commonly is described as Kerr lensing and self-phase modulation. Reduction of this negative influence usually implies decrease of effective optical pulse intensity which can be quantified in terms of so-called $B$ integral, in essence representing nonlinear on-axis phase shift (Brawn, 1981). In case of regenerative amplification multiple passes should be taken into account in order to evaluate total $B$ integral accumulated during amplification stage:

$$
B \approx B_{1} \int_{0}^{\tau} \varepsilon\left(t^{\sim}\right) d t^{\sim},
$$

where $B_{1}$ is single pass $B$ integral calculated for the intensity which is equal to the gain media saturation fluence divided by the pulse duration (see the Appendix section for details). The energy integral in this formula exhibits a factor of effective impact of multiple passes. We have already met such an integral when evaluated total lost part of the energy (see Eq. 14) and the value proportional to this factor has been depicted in previous section in Fig. 8. We can see that operation at the upper branch of the separatrix with short pulses is strongly unadvisable since the $B$ integral is increased by several times. The cause of that is obviously multiple passes of intense pulses peculiar in a regime behind the instability zone. That concerns the $B_{1}$ value, its reduction, in a condition of fixed pulse duration, simply implies standard methods of the mode area increase and shortening of the volume intracavity components. However these possibilities are rather limited. Even so the thin disc geometry allows tremendous reduction of gain media length (Speiser \& Giesen, 2008) but the Pockels cell still can exhibit a real challenge. Among known to date Pockels cell materials only the BBO crystal is suitable for high average power (due to low absorption) and for high repetition rates (thanks to relatively low acoustic ringing). However the transverse electro-optic effect, the only functional for this crystal, permits shortening the optical pass or aperture increasing only in limited extent until driving voltage becomes unacceptably high (Nickel et al., 2005). Thus, since the Kerr effect often restricts the capabilities of regenerative amplifiers it is important at least to select correctly the operation regime in order to minimize its impact. We can note that operation at higher seed energy $\left(\varepsilon_{s}\right.$ larger than ample seed pulse energy) is beneficial in this respect too. At that condition, operation at maximum output ( $\tau_{\max }$ curve) does not suffer from too high multiple pass factor of the $B$ integral [see Fig. 8(b)]. 


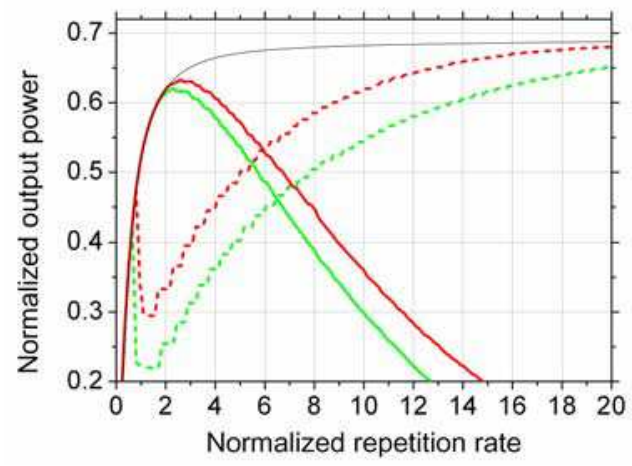

Fig. 10. Theoretical output power versus repetition rate for the threshold gain of 0.028 and normalized seed pulse energy of $\varepsilon_{s}=7.7 \times 10^{-7}$ (red line) and $\varepsilon_{s}=7.7 \times 10^{-9}$ (green line). The data of high and low separatrix branches correspond to solid and dashed lines respectively. The black line is the reference curve of achievable power.

At the end of the theoretical part of the chapter we are giving diagrams of the basic performance parameter - maximum output power which can be achieved in a condition of stability maintaining $\left(\tau=\tau_{\text {opt }}\right)$, Fig. 10. Presented normalized average power is defined as a product of normalized energy and normalized repetition rate. The power curve, calculated for $\tau_{\max }$ round trip number, can be regarded as the reference curve corresponding to theoretical efficiency limit. Really, in accordance with the $\tau_{\max }$ definition, the corresponding output power is the highest obtainable average power in assumption of period doubling absence. The calculations show that this power is invariant under the seed energy, although the $\tau_{\max }$ itself is not. Achieving the calculated maximum average power implies the best possible utilization of the stored pump energy, i.e. it assures the highest power efficiency. Obviously the same top performance we can reach operating at the ample seed energy provided that the round trip number is set equal to $\tau_{\max }$. This attribute is the main benefit of the high seed pulse energy at high repetition rates. The power defect with respect to the reference power curve observed for lower seed energies is essentially caused by instability effects. We present curves related to both branches of the separatrix within critical repetition rate range. The data related to the upper branch are not always reliable because as we have shown earlier this regime suffers from indirect effects related to multiple passes of intense optical pulse such as the Kerr effect and excessive heating of optical components.

In concluding this theoretical part, now we are ready to proceed to experimental verification of the ideas developed above.

\section{Amplification experiments}

\subsection{Experimental setup}

The amplification experiments were carried out in order to demonstrate basic features of amplification dynamics and to verify theoretical results presented above. The knowledge of potential capabilities and of general limitations makes it possible to provide the best regime selection and deliberate optimization of control parameters. In practicality, this means to maximize extraction of the given stored population inversion as a stable train of output 
pulses. We leave outside the scope of present consideration optimization of the pump characteristics and the geometry of the optical resonator allowing more power in TEM $\mathrm{M}_{00}$ mode, as these do not relate directly to the amplification dynamics. The parasitic intracavity losses, although formally a governing parameter, are not an object of consideration; they should simply be reduced as much as technically possible. Since the repetition rate is usually imposed by the specifications it appears as a variable but a given parameter. There are two adjustable parameters which can be used for the system optimization - the number of roundtrips and the seed pulse energy.

We performed experiments with a system based on $\mathrm{Nd}: \mathrm{YVO}_{4}$ crystals, a gain medium with truly four-level nature (except terminal-level nuances). The schematic diagram of the experimental setup that we used for investigation of regenerative amplification is shown in Fig. 11. In essence the system consists of the seed source and the regenerative amplifier itself.

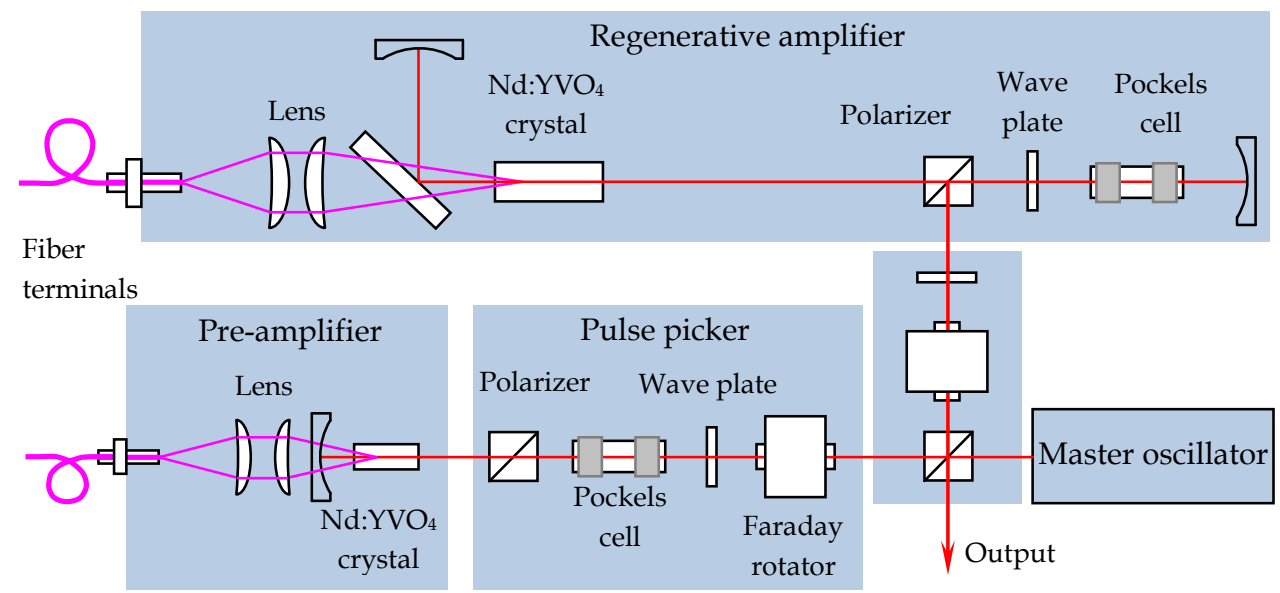

Fig. 11. Schematic diagram of the experimental setup.

The seed pulse source for regenerative amplification experiments was based on a diodepumped passively mode locked picosecond $\mathrm{Nd}: \mathrm{YVO}_{4}$ laser of moderate power. It generated a continuous pulse train with repetition frequency of $82 \mathrm{MHz}$ and average power of $300 \mathrm{~mW}$. The laser was able to produce optical pulses with duration as short as $6 \mathrm{ps}$. The short pulses were used in experiments where dynamics peculiar to high peak intensities were of interest. The initial investigations were focused on the "pure" dynamics not disturbed by optical nonlinearities. These experiments were carried out with 58 ps duration pulses obtained by installing an etalon in the oscillator cavity (the etalon narrows the bandwidth, thus widening the pulse duration).

A pulse picker was used to select pulses for further amplification and in this way to control the effective repetition frequency of the seed source. This part of the seed source system is important for high repetition rate operation, especially based on high gain laser media like neodymium doped vanadates. If the pulse picker is not used then two negative effects caused by unwanted pulses have place. These pulses continuously pass the optical resonator of the regenerative amplifier during pump stage and go out spatially coinciding with useful output signal. This leads first to reduced pulse contrast and second to parasitic consumption 
of stored energy. One can decrease the seed pulse energy and compensate for that by increasing number of round trips. This simple approach may often avoid bad influence of the unwanted seed background while operating at low repetition rates. The reduction of the seed energy, as we have already seen (theoretically), is not a good idea when turning operation to high repetition rates. In our setup the pulse picker was an electro-optic switch based on an RTP Pockels cell. Selected pulses formed an input signal for the preamplifier. The remaining pulses of the master oscillator train were directed to the fast photodiode for synchronization of electro optic components of the system including the pulse picker itself.

A double pass $\mathrm{Nd}: \mathrm{YVO}_{4}$ preamplifier installed behind the pulse picker was used to increase the seed pulse energy to required ample energy. High emission cross section of the $\mathrm{Nd}: \mathrm{YVO}_{4}$ crystal make this system efficient at relatively low input average power. Only $2 \mathrm{~W}$ of pumping was sufficient to achieve a gain coefficient of more than two orders of magnitude. The seed pulse energy was $3.2 \mathrm{~nJ}$ when pumping of the preamplifier was switched off. The energy of the pre-amplified pulse reached $1.1 \mu \mathrm{J}$ at $10 \mathrm{kHz}$ and steadily decreased with the repetition rate to $370 \mathrm{~nJ}$ at $200 \mathrm{kHz}$. The calculation presented in the next section will show that the obtained energy is sufficient to ensure stable operation.

Simple estimations show that the preamplifier is a good alternative in comparison with a more straightforward seed source scheme based on a powerful master oscillator. In order to provide $370 \mathrm{~nJ}$ pulses the master oscillator operating in a CW mode locking regime with a reasonable repetition frequency of $50 \mathrm{MHz}$ should generate average power of $18.5 \mathrm{~W}$. This way is really prodigal since the useful part of this power is much lower, e.g. only $74 \mathrm{~mW}$ even operating at $200 \mathrm{kHz}$. Obviously the preamplifier is a much more energy-efficient solution.

The regenerative amplifier was comprised of an optical resonator containing the gain medium (Nd: $\mathrm{YVO}_{4}$ crystals) and an electro-optic switch. The electro-optic switch consisted of a BBO Pockels cell, a quarter-wave plate and a thin-film polarizer. The total multi-pass gain of the regenerative amplifier depends on the number of cavity round trips which is determined by the amplification-stage duration. This important parameter is easily controlled by setting the time interval during which the high voltage is applied to the Pockels cell.

The laser crystal was continuously pumped by the fiber coupled laser diode module with fiber core diameter of $400 \mu \mathrm{m}$ and numerical aperture of 0.22 . Optimal pump power, providing maximum output in $\mathrm{TEM}_{00}$ mode, was set to be $44 \mathrm{~W}$. This optimization was performed in CW generation mode. Provided that no voltage was applied to the Pockels cell and the quarter-wave plate was adjusted for maximum output (optimal output coupling conditions), $12.5 \mathrm{~W}$ of average power was obtained.

The output radiation was diverted from the input signal path by standard optical circulator based on Faraday rotator. The repetition rate of the system was limited to $200 \mathrm{kHz}$ by electronics driving the electro-optic switches.

\subsection{Application of stability diagrams to amplification experiments}

Now we can apply the concepts developed in the theoretical part to estimating behavior of a real system. At the beginning the basic system properties should be evaluated in order to perform reciprocal transformation of normalized parameters to dimensional ones corresponding to real operation conditions. The steady state small signal gain, determined by the pump intensity, was found directly as the ratio of the seed energies measured right 
before and behind the active element of the regenerative amplifier. The parasitic intracavity losses were derived from the specifications of the optical components. The laser characteristics of $\mathrm{Nd}: \mathrm{YVO}_{4}$ crystal (emission cross section and gain relaxation time) were taken from (Peterson et al., 2002). We explored amplification of three seed pulse energies differing by about two orders of magnitude: pre-amplified seed, unamplified seed and attenuated seed. This set of input signals covers the functionally important range. The value of the unamplified seed, $3.2 \mathrm{~nJ}$, is of the same order of magnitude as the pulse energy of commonly used moderate power solid-state picosecond lasers. Operation with the seed energy intentionally attenuated to $32 \mathrm{pJ}$ provides opportunity to evaluate typical behavior of the regenerative amplifier seeded with potentially attractive low power sources, e.g. with ultrafast laser diodes, which would substantially reduce system size and complexity. The seed energy obtained with the pre-amplifier was expected to be high enough to reach ample level. These seed energies were measured at the output of the seed formation system. However we observed that during further amplification the seed energy was not completely exploited. Mode mismatching reduces the effective seed energy. In general, it is difficult to avoid mode mismatching between a seed laser and the optical cavity of the regenerative amplifier in both spatial and spectral domains. Spectral mismatching can exist even with identical gain media because of e.g. different temperatures of laser crystals in those devices (Murray \& Lowdermilk, 1980).

\begin{tabular}{|l|l|}
\hline \multicolumn{1}{|c|}{ Parameter } & \multicolumn{1}{c|}{ Value } \\
\hline Wavelength & $1064 \mathrm{~nm}$ \\
\hline Emission cross section & $11.4 \times 10^{-19} \mathrm{~cm}^{2}$ \\
\hline Gain relaxation time & $83 \mu \mathrm{s}$ \\
\hline Effective mode diameter in the laser rod & $1 \mathrm{~mm}$ \\
\hline Steady state small signal gain & 2.94 \\
\hline Seed pulse energies & $11 \mathrm{pJ}(\mathrm{low}) ; 1.1 \mathrm{~nJ}$ (medium); 240 nJ (high) \\
\hline
\end{tabular}

Table 2. Parameters used for stability diagrams calculation

In order to have appropriate data for theory verification we experimentally estimated the overall level of mismatching. We measured real double pass small signal gain of the whole regenerative amplifier (output/input energy ratio while the Pockels cell was disabled) and compared this value with "pure" steady state gain of the laser element. Thus we determined the effective seed value to be about three times less than the measured one. The primary parameters of the regenerative amplifier which we used for calculations are summarized in Table 2.

Before proceeding to stability diagrams describing our particular experimental conditions, we note that we utilized a linear cavity that establishes double pass through the gain media, while the modeling has implied single pass for one cavity round trip. This factor of two was taken into account so that the $y$-axis of stability diagrams corresponds to real round trip number, $t / T_{0}=1 / 2 \tau / G_{0}$. The other relations between dimensional and normalized parameters remain unchanged as they have been given in the section 2.2.

The stability diagrams describing amplification experiments are presented in Fig. 12. These data were obtained in the approximation of short pulses (accounting for terminal-level lifetime effect). As we have already known, the system capabilities are completely exploited when the $\tau_{\max }$ curve is outside the instability region. The diagrams show that at low seed energy (11 pJ) the appropriate repetition rates should be less than $20 \mathrm{kHz}$. For the medium 
seed level $(1.1 \mathrm{~nJ})$ this range increases to $25 \mathrm{kHz}$. At higher repetition rates the $\tau_{\max }$ curve enters the instability zone. It is important that for $240 \mathrm{~nJ}$ or higher seed energies the $\tau_{\max }$ curve does not enter the instability zone in the whole range of repetition rates. So, for our laser system this energy corresponds to the ample seed value, sufficient to eliminate negative features of amplification dynamics, and thus to completely exploit the system capabilities.
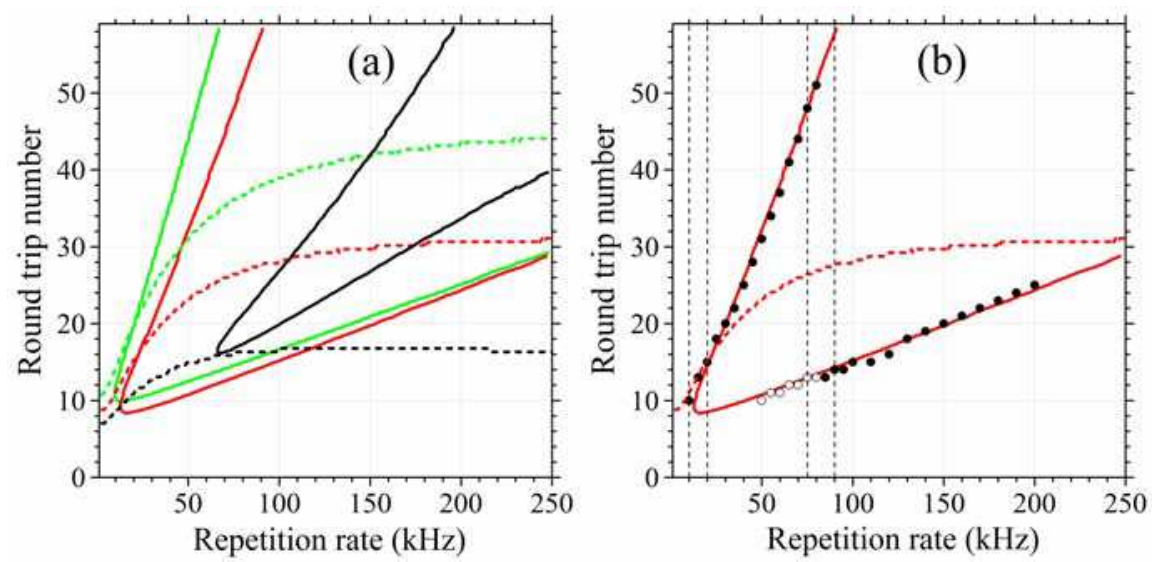

Fig. 12. (a) Separatrix curves (solid lines) and $\tau_{\max }$ curves (dotted lines) in parameter space. Black, red and green lines correspond to seed pulse energies of $240 \mathrm{~nJ}, 1.1 \mathrm{~nJ}$ and $11 \mathrm{pJ}$, respectively. (b) Operating point trajectories (vertical dashed lines) and measured number of optimal round trips for the pulse durations of 58 ps (solid circles) and 9 ps (open circles) with respect to stability diagram for the seed energy of $1.1 \mathrm{~nJ}$.

\subsection{Experimental bifurcation diagrams}

The initial experiments were carried out with medium seed pulse energy (the preamplifier was disabled). Various dynamic regimes, depending on set of control parameters, were observed. As an illustration, Fig. 13 shows oscilloscope screen shots of the output pulse train in typical single-energy and period doubling regimes.
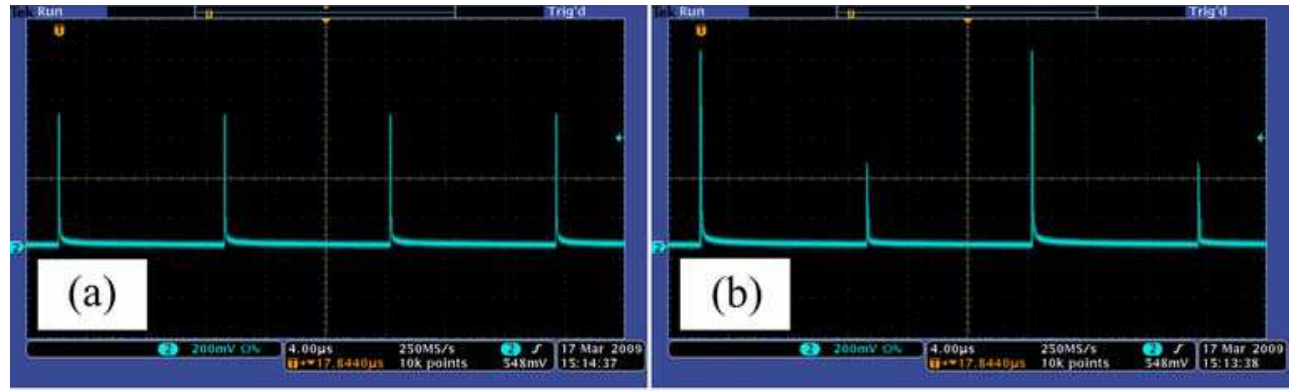

Fig. 13. Screenshots of typical pulse trains at $90 \mathrm{kHz}$. Stable energy output and the $2 \mathrm{~T}$ period doubling regime were obtained at number of round trips equal to 14 (a) and 16 (b) respectively. 
Experimentally obtained diagrams of the average output power and pulse energy versus number of round trips demonstrating system behavior at different repetition rates are presented in Fig. 14. The specific repetition rates were chosen to describe the most relevant cases of regenerative amplifier dynamics in respect of system optimization. The singlepeaked dependence inherent to low repetition rates appears at $10 \mathrm{kHz}$ [Fig. 14(a)]. The average power and the pulse energy reach the maximum values simultaneously, when the round trip number is equal to ten. At $20 \mathrm{kHz}$ the situation is different [Fig. 14(b)]. The shape of the energy curve shows that the system undergoes bifurcation in the 9-13 range of the round trip numbers. However, in this case the period doubling does not affect the system performance because the output power reaches its maximum value in a single-energy regime. This repetition rate is still not critical.

Instability effects become more pronounced at higher pulse repetition rates. The period doubling not only breaks the energy stability but also distorts the curve of the average power (as described in section 2.8). This curve now has two explicit peaks [Figs. 14(c) and 15(d)]. The first peak, corresponding to the maximum power, is located in a period doubling zone, whereas the second one is just over the instability edge. The optimal regime is obtained in the vicinity of the bifurcation point. At $75 \mathrm{kHz}$ the optimal number round trips is equal to 48 . This point is close to the second power peak, on the right side of the period doubling zone [Fig. 14(c)]. For $90 \mathrm{kHz}$ repetition rate the optimal number round trips is equal to 13 and is situated right before the first bifurcation point [Fig. 14(d)].

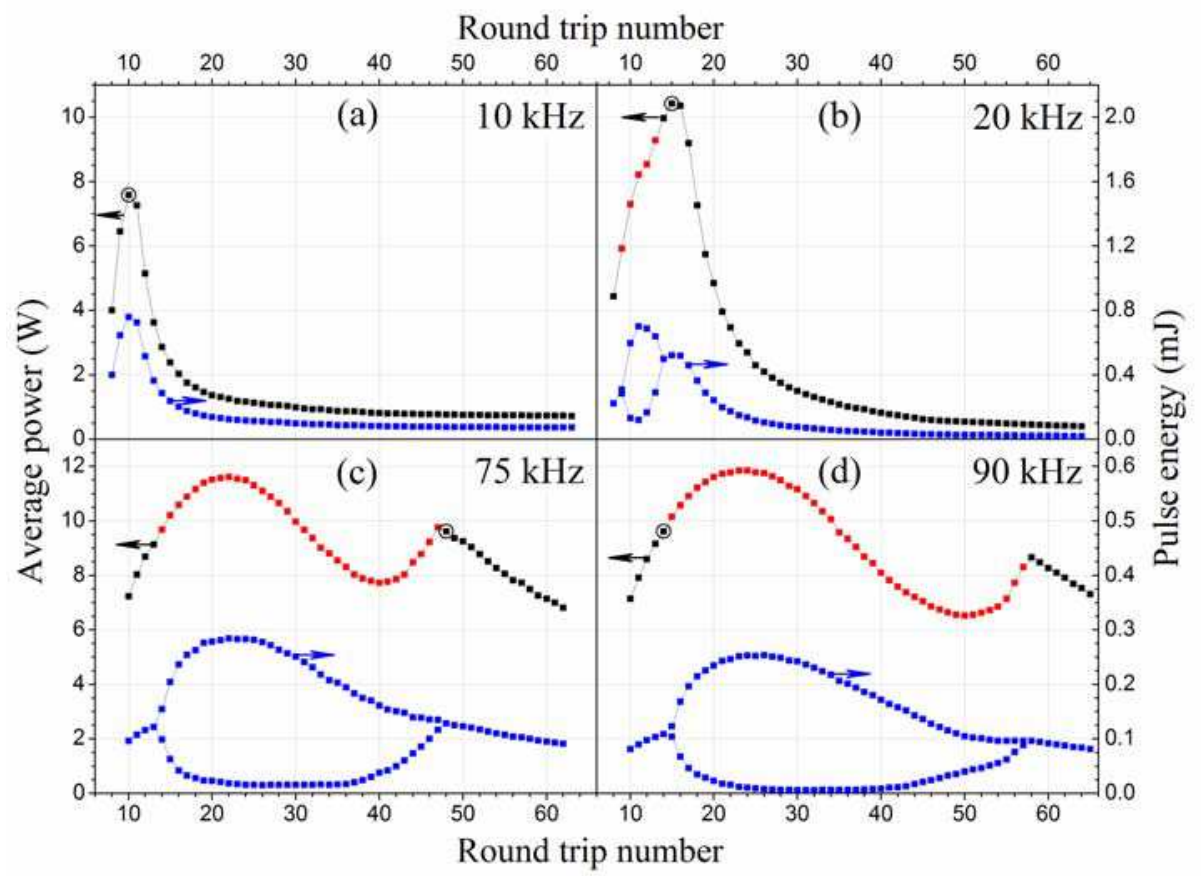

Fig. 14. Experimental average power (black and red dots correspond to stable and unstable regimes respectively) and pulse energy (blue dots) versus number of cavity round trips for the selected repetition rates. The encircled points correspond to the maximum power at stable operation. 
The trajectories of the operating points corresponding to variation round trip number at a constant repetition rate are presented in Fig. 12(b). This trajectory at $10 \mathrm{kHz}$ does not pass the instability zone. At $20 \mathrm{kHz}$ the optimal operating point is above the instability zone. Both repetition rates $75 \mathrm{kHz}$ and $90 \mathrm{kHz}$ are critical - the optimal number of round trips is on the stability edge and is rather far from the point of the highest attainable power. The experimentally observed results confirm theoretical predictions that for critical repetition rates: (i) the output energy exhibits unacceptable fluctuations when the amplifier produces the highest average power, (ii) the highest stable pulse energy is reached close to the instability edge.

Some effects caused by nonzero terminal-level lifetime were also observed in the experiment. The presented bifurcation diagrams at $75 \mathrm{kHz}$ and $90 \mathrm{kHz}$ theoretically should exhibit not only $2 T$ but also $4 T$ regimes [like in Fig. 6(e)], if the long pulse approximation is applied. The regime of $4 T$ period doubling was not observed experimentally and it should not exist theoretically, provided that contribution of the terminal-level lifetime is accounted. At the same time, as the theory has predicted, terminal-level lifetime effect does not influence the system performance at such low seed level.

Real deviation from theory is observed at the lowest repetition rate $(10 \mathrm{kHz})$. The output energy decays too fast behind the peak point in comparison with theoretical expectations [see Fig. 6(b)]. This occurred because of the Kerr effect influence was substantial at low repetition rates even for initial experiments performed with relatively long 58 ps pulse duration.

\subsection{Performance evaluation and discussion}

It has been shown in the theoretical part that variation of only roundtrip number does not solve the stability problem; increase in the seed pulse energy is required in order to avoid bifurcations and corresponding instability at high repetition rates. So, we proceed to experimental verification of the seed energy influence. We compared operation for three cases: "low", "medium", and "ample" pulse seed energy.

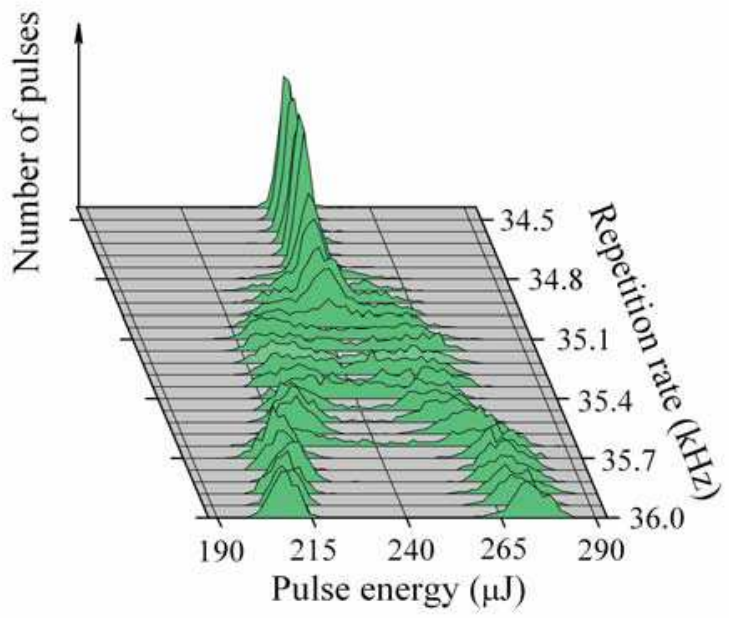

Fig. 15. Experimental energy histogram in the vicinity of the bifurcation point. 
The measurements were performed at optimal round trip numbers. They were set for maximum average power while maintaining stable operation for every repetition rate. The formulation of stability criteria in real experimental environments requires certain attention. Experimental discrimination of amplification regimes to some extent suffers from uncertainty because of technical instabilities, not related to fundamental system properties. Technical noises, in essence slight modulation of governing parameters usually with random distribution, limit system stability. The most typical of these are pumping source noises, seed pulse energy fluctuation, synchronization jitter, resonator disturbance by mechanical vibrations and by air flow. In our setup the technical, not disturbed by period doubling, standard deviation of output energy was less than $0.7 \%$. In proximity to a bifurcation point the deviation increased. The diagram of typical transition from single energy to period doubling regime "under magnification" of the repetition rate scale is shown in Fig. 15; the bifurcation point looks rather as a spot than as a point. The uncertainty takes place in the range where the deviation of energy is clearly higher than the technically conditional level but where the two peaks are still not distinguishable. In the repetition rate scale this range does not exceed $1 \mathrm{kHz}$ (typically the value is $0.5 \mathrm{kHz}$ ). In the round trips scale measurements with such fine steps are not possible (in contrast to theoretically grounded terms the real experimental round trips are discrete), and besides, the analysis of the energy histogram is a time consuming procedure. So, we used simple phenomenological criterion formulated for the particular setup allowing real time measurements. The operation was considered stable when the standard deviation of the pulse energy did not exceed 1\%. Apart from that, special care was taken regarding origin and spectrum of disturbing noises to be sure that they are virtually non-periodic. In particular the pulse picker driving electronics had to be improved in order to eliminate seed train modulation at the frequency equal to half the system repetition rate. We can note that even barely perceptible presence of "resonant" components (sub-harmonics of dumping frequency) in the spectrum of technical noises may tremendously enhance the influence of period doubling and can change the dynamical pattern beyond recognition.
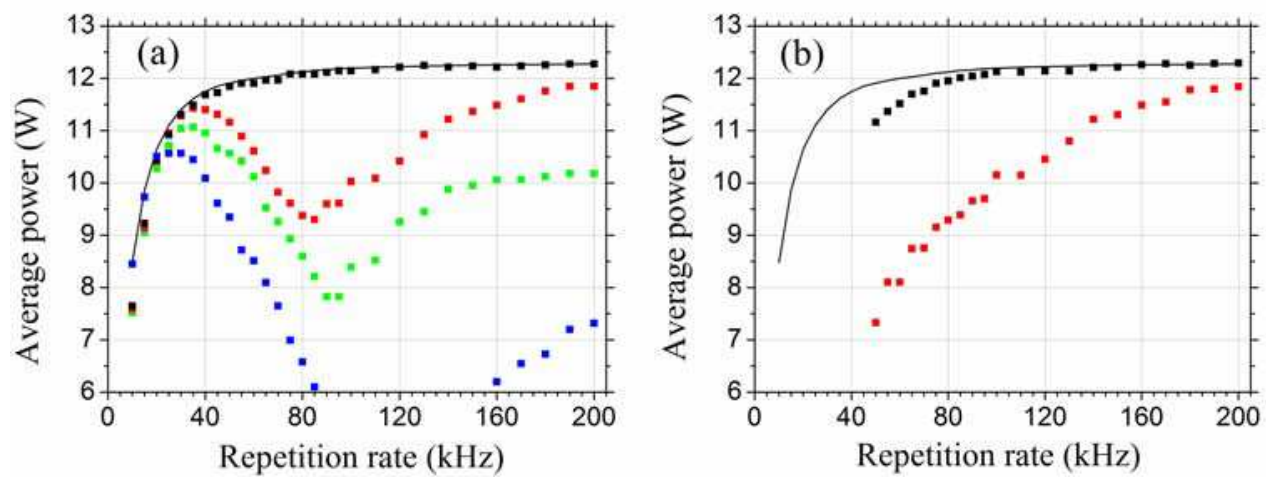

Fig. 16. Experimental output power versus repetition rate for 58 ps (a) and 9 ps (b) pulses. Black, red and green dots correspond to measured seed pulse energies of $700 \mathrm{~nJ}, 3.2 \mathrm{~nJ}$ and $32 \mathrm{pJ}$, respectively. Theoretical curve of achievable power is solid line in both diagrams. Blue dots in Fig. 16(a) are Q-switch experiment results. 
Experimental dependences of the average output power versus repetition rate are presented in Fig. 16(a). The output is virtually independent of the seed level for low repetition rates. At higher rates there is a drop in power for low and medium seed levels in compliance with theoretical notions. The most significant power decrease appears in the $80-95 \mathrm{kHz}$ range, then the output power steadily grows as the repetition rate increases.

Non-monotonic behavior of the power curve originates from the specific location of optimal operation points in the parameter space. Corresponding experimental data with respect to the theoretical stability diagram for medium seed energy is presented in Fig. 12(b). The operating points coincide with the theoretical curve of $\tau_{\max }$ until the latter enters into the instability zone (at $25 \mathrm{kHz}$ ). Then operation at the upper branch of the separatrix becomes optimal. However as the repetition rate increases the stable operating point moves further away from the $\tau_{\max }$ position, resulting in lower output energy. Consequently, starting from $85 \mathrm{kHz}$ the optimal operation point switches to the lower border of the instability region. In this regime the operating point gradually comes closer to the $\tau_{\max }$ position, and consequently, the output power steadily increases with increasing repetition rate.

The pre-amplified seed pulse has sufficient energy to maintain stable operation at maximum power, in accordance with ample seed properties. The power curve obtained with the preamplifier shows no signs of downward excursion with respect to the reference curve (the curve of theoretically attainable power corresponding to $\tau_{\max }$ round trip number). Some slight deviation observed at low repetition rates was assumed to be caused by the Kerr effect. This nonlinearity can cause additional intensity-dependent intracavity losses (more pronounced at higher pulse energies), and so its influence is more pronounced at lower repetition rates. In order to differentiate the Kerr effect influence we performed experiments with nanosecond pulses in the same setup. The seed source was disabled so that the regenerative amplifier was transformed to a Q-switched laser with cavity dumping. Basically this device can be regarded as a regenerative amplifier seeded by spontaneous emission getting into the lasing mode. Thus, the seed energy becomes extremely low and additionally the laser system turns to the nanosecond domain $(\approx 15 \mathrm{~ns}$ output pulse length was obtained in our setup). The former gives a large drop in power at critical repetition rates, the latter gives enhanced, with respect to the regular regenerative amplification, output energy at low repetition rate (the Kerr effect is negligible for such long pulses). The nanosecond pulse energy at low repetition rates exceeds the picosecond energy and very well agrees to the theoretical curve [Fig. 16(a)]. This demonstrates that experimental deviations appeared because of the Kerr effect and consequently general validates that the theoretical approach we have developed is wholly satisfactory.

Heating of intracavity components was not observed to affect regenerative amplifier operation in optimal round trip number experiments. However, clear evidence of excessive heating was noticed during recording of bifurcation diagrams in the worse (from this point of view) regime. The beam quality was observed to deteriorate for $90 \mathrm{kHz}$ repetition rate and at round trip numbers between 46 and 56. From the other hand the average power defect reached maximum in this range [see Fig. 14(d)]. As we have concluded theoretically, this defect is indeed due to dissipated power, the absorbed fraction of which is heating intracavity optics.

We also compared performances of the regenerative amplifier seeded by high and medium pulse energy for the functionally important case of shorter optical pulses. Typically about 9 ps pulse duration was obtained at the output of the amplifier seeded by the 6 ps pulse. 
This duration is close to the minimum value supported by the gain bandwidth of the $\mathrm{Nd}: \mathrm{YVO}_{4}$ crystal in high total-gain applications such as regenerative amplification. The measurements were constrained to dumping rates above $50 \mathrm{kHz}$. Nevertheless, the intensities were substantial, and the Kerr effect influence was so strong that it eventually resulted in decrease of the output power [Fig. 16(b)]. The average power obtained with the pre-amplified seed was slightly lower than that theoretically predicted below $80 \mathrm{kHz}$ and the difference reached $6.7 \%$ at $50 \mathrm{kHz}$. However, the comparison of these characteristics with those obtained at a medium seed level shows that the benefit of the preamplifier is even more pronounced in case of shorter pulses. The difference is related to a large decrease of the average power below $85 \mathrm{kHz}$ for the case of the medium seed. This is direct consequence of inefficient operation at the upper separatrix branch in critical repetition rate range under the Kerr effect influence. Experimental optimal operation points for both 58 ps and 9 ps pulses with respect to theoretical stability diagram are represented in Fig. 12(b). The optimal operating points for the short pulse experiments were always settled along the less efficient lower branch of the parameter separatrix. Attempts to operate at the upper branch (optimum for long pulses) resulted in an even larger decrease of the output. In order to quantify this difference we estimated the multi-pass $B$ integral of the system (Eq. 22 in the Appendix). The $B$ integral calculated at $50 \mathrm{kHz}$ repetition rate gave values of 1.3 (acceptable) and 7.6 (problematic) at the transition from low to upper separatrix branch respectively. Thus the high seed energy gives additional advantage at shorter pulses due to a significantly lower value of the optimal number of round trips.

The amplification experiments which were performed with $\mathrm{Nd}: \mathrm{YVO}_{4}$ regenerative amplifier have shown that the developed theoretical approach accurately agrees with experimental data and can be used for practical system-design guidelines.

\section{Conclusions}

Continuously pumped regenerative amplifiers are subject to energy instability at high pulse repetition rates due to period doubling bifurcation. Theoretical concepts representing a generalized picture of operation features have been in-detail worked out in order to differentiate and understand instability effects. Experimental data for $\mathrm{Nd}: \mathrm{YVO}_{4}$ regenerative amplifier have been presented; and possible techniques for performance optimization have been analyzed. An increase in the seed pulse energy has been demonstrated to improve amplification dynamics. Addition of a preamplifier is shown to be not only a convenient means for regenerative amplification investigation at a wide range of seed energies but also an efficient way to get top performance in practice. The $\mathrm{Nd}: \mathrm{YVO}_{4}$ preamplifier delivered seed energy high enough to provide stable operation at repetition rates up to $200 \mathrm{kHz}$ with average output power near the theoretical limit.

We have not performed appropriate experiments to elaborate amplification dynamics for ytterbium based regenerative amplifiers. However, it would be interesting to verify theoretical predictions for ytterbium doped crystals as for media exhibiting pronounced quasi-three-level behavior. Especially, because similar types of crystals may exhibit different sensitivity to bifurcations in the same setup, as reported by (Buenting et al., 2009) and (Sayinc et al., 2009). In addition, bulk ytterbium doped materials are low gain materials and therefore the preamplifier technique is not so easily applicable to such systems. There is demand to create another method to keep regenerative amplifiers stable at maximum output power, possibly some kind of feedback. Such an idea was formulated by (Dörring et 
al., 2004) but as far as we know neither theoretical modeling nor practical realization of this approach has been reported so far. Essentially, this can be an attempt to stabilize the inherently unstable balance between pumping and inversion depletion - that is constant forced return of the system state to the originally repulsive fixed point. Actually the initial gain of amplification phase should be kept constant by the feedback. However, straightforward engineering does not work in this case because output energy signal of the current operation cycle does not contain sufficient information to adequately control pump power of the subsequent cycle. So elaboration of this problem can be a goal for further theoretical research and experimental work.

\section{Appendix. Multi-pass $B$ integral for regenerative amplifier}

The conventional quantitative gauge of the Kerr effect in laser systems is the $B$ integral, nonlinear on-axis phase shift which light waves with wavelength $\lambda$ undergo propagating through the media:

$$
B_{s p}=\frac{2 \pi}{\lambda} \int n_{2}(z) I(z) d z .
$$

The terms $I(z)$ and $n_{2}(z)$ are distributions of on-axis intensity and nonlinear refractive index along current coordinate $z$. In order to evaluate the Kerr effect accumulated during regenerative amplification the integration should be performed over all the roundtrips of the optical cavity (multi-pass $B$ integral). Then the full integration length is a product of the optical cavity pass length and round trip number. In an approximation of relatively low single pass gain (the integral within the gain medium can be replaced with the average) and also for moderate Kerr effect influence (iteration of the intensity profile in the optical resonator is not disturbed too much by self focusing) we can replace the overall integral with a sum of single pass integrals:

$$
B \approx \frac{2 \pi}{\lambda} \sum_{N R T} \int n_{2}(z) I_{N R T}(z) d z,
$$

where index NRT implies summation over round trips. The sum of integrals is equal to the integral of the sum and also $n_{2}(z)$ is independent of round trip number function, then we obtain:

$$
B \approx \frac{2 \pi}{\lambda} \int n_{2}(z) \sum_{N R T} I_{N R T}(z) d z
$$

In an assumption of Gaussian beam shape for which the peak intensity is equal to $2 P /\left(\pi w^{2}\right)$ (the term $P$ is the optical power, the term $w$ is the Gaussian beam radius) we can calculate the intensity for the pulse duration $\Delta t$ in terms of pulse energy:

$$
\sum_{N R T} I_{N R T}(z) \approx \frac{2}{\pi w^{2}(z) \Delta t} \sum_{N R T} E_{N R T} .
$$

Summation of energies can be rewritten as time integration, and then dimensional current energy can be represented in terms of normalized energy which we have introduced in section 2.2: 


$$
\sum_{N R T} E_{N R T} \approx \frac{1}{T_{0}} \int_{0}^{N R T \cdot T_{0}} E(t) d t=\frac{A_{a} F_{s a t} G_{0}}{T_{0}} \int_{0}^{N R T \cdot T_{0}} \varepsilon(t) d t=A_{a} F_{s a t} \int_{0}^{\tau} \varepsilon\left(t^{\sim}\right) d t^{\sim} .
$$

At that, the limit of integration NRT. $T_{0}$ (overall pulse propagation time) is reducing to the effective round trip number $\tau$ when the integration variable, current dimensional time $t$, has been transformed to the normalized time $t^{\sim}$. Also, the laser material properties, responsible for stimulated emission, are combined to a conventional macroscopic term, saturation fluence, the ratio of photon energy and stimulated emission cross section: $F_{\text {sat }}=h \omega / \sigma$. The beam area in the active medium obviously can be expressed through the Gaussian beam radius: $A_{a}=\pi w_{a}^{2}$. And finally, using straightforward transformation and normalized energy integral calculated in section 2.8 (Eq. 14), we derive explicit expression of $B$ integral, the diminished form of which has been used in section 2.9 (Eq. 16):

$$
B=B_{1} \int_{0}^{\tau} \varepsilon\left(t^{\sim}\right) d t^{\sim} \text {, where } B_{1}=\frac{4 \pi}{\lambda} \frac{F_{s a t}}{\Delta t} \int n_{2}(z)\left[\frac{w_{a}}{w(z)}\right]^{2} d z \text { and } \int_{0}^{\tau} \varepsilon\left(t^{\sim}\right) d t^{\sim}=\frac{g_{a f}-g_{a i}-\varepsilon_{f}}{g_{t}} .
$$

We have obtained the multi-pass $B$ integral for evaluation of the Kerr effect in regenerative amplifiers as the product of two factors. The first factor, $B_{1}$ represents attributes of the system geometry, material parameters and optical pulse duration. Essentially, this term is single-pass $B$ integral calculated for the Gaussian beam in given optical cavity and for pulse energy fluence equal to the gain medium saturation fluence. The second factor is a function of regenerative amplifier regime represented in normalized terms (the dissipated energy divided by the threshold gain). This is convenient for practical application form in which functional physical contributions are separated.

\section{Acknowledgements}

The authors wish to acknowledge the technical assistance of Juozas Verseckas from EKSPLA $\mathrm{UAB}$ in preparation of the experimental setup, Vidmantas Gulbinas from Institute of Physics and Lucian Hand from Altos Photonics Inc. for fruitful discussions of the manuscript. This work was partially financed by the Eurostars Project E!4335-UPLIT.

\section{References}

Alligood, K; Sauer, T. \& Yorke, J. (1996) Chaos. An Introduction to Dynamical Systems, Springer-Verlag, ISBN 0-378-94677-2, New-York

Arecchi, F.; Meucci, R.; Puccioni, G. \&. Tredicce, J (1982) Experimental Evidence of Subharmonic Bifurcations, Multistability, and Turbulence in a Q-Switched Gas Laser. Phys. Rev. Lett., Vol. 49, Issue 17 (October 1982), 1217-1220, ISSN 0031-9007

Bibeau, C.; Payne, S.; \& Powell, H. (1995) Direct measurements of the terminal laser level lifetime in neodymium-doped crystals and glasses. J. Opt. Soc. Am. B, Vol. 12, No. 10 (October 1995), 1981-1992, ISSN 0740-3224

Biswal, S.; Itatani, J.; Nees, J. \& Mourou, G. (1998) Efficient energy extraction below the saturation fluence in a low-gain low-loss regenerative chirped-pulse amplifier. IEEE J. Sel. Top. Quantum Electron., Vol. 4, Issue 2 (March 1998), 421-425, ISSN 1077$260 X$ 
Brawn, D. (1981) High Peak Power Nd:Glass Laser Systems, Springer-Verlag, ISBN 0387105166, New-York

Buenting, U.; Sayinc, H.; Wandt, D.; Morgner, U.; \& Kracht, D. (2009) Regenerative thin disk amplifier with combined gain spectra producing $500 \mu \mathrm{J}$ sub $200 \mathrm{fs}$ pulses. Opt. Express, Vol. 17, No. 10 (May 2009), 8046-8050, ISSN 1094-4087

Clubley, D.; Bell, A. \& Friel, G (2008) High average power Nd:YVO based pico-second regenerative amplifier. Proc. SPIE, Vol. 6871 (February 2008), 68711D, ISSN 0277$786 \mathrm{X}$

Dörring, J.; Killi, A.; Morgner, U.; Lang, A.; Lederer, M. \& Kopf, D. (2004) Period doubling and deterministic chaos in continuously pumped regenerative amplifiers. Opt. Express, Vol. 12, No. 8 (April 2004), 1759-1768, ISSN 1094-4087

Fermann, M.; Galvanauskas, A. \& Sucha G. (2002) Ultrafast Lasers: Technology and Applications, Marcel Dekker, ISBN 0-203-91020-6, New York

Forget, S.; Balembois, F.; Georges, P. \& Devilder P. (2002) A new 3D multipass amplifier based on Nd:YAG or Nd:YVO 4 crystals. Appl. Phys. B, Vol. 75, No. 4-5 (October 2002), 481-485, ISSN 0946-2171

Grishin, M.; Gulbinas, V. \& Michailovas, A. (2007) Dynamics of high repetition rate regenerative amplifiers. Opt. Express, Vol. 15, Issue 15 (July 2007), 9434-9443, ISSN 1094-4087

Grishin, M.; Gulbinas, V.; Michailovas, A. \& Verseckas, J. (2008) Operation Features of Regenerative Amplifiers at High Repetition Rate, Technical digest in $C D$, paper CFB7, Conference on Lasers and Electro-Optics, CLEO-08, San-Chose, CA, USA, May 4-9, 2008, OSA

Grishin, M.; Gulbinas, V. \& Michailovas, A. (2009) Bifurcation suppression for stability improvement in $\mathrm{Nd}: \mathrm{YVO}_{4}$ regenerative amplifier. Opt. Express, Vol. 17, Issue 18 (August 2009), 15700-15708, ISSN 1094-4087

Haken, H. (1975). Analogy between higher instabilities in fluids and lasers. Physics Letters A, Vol. 53, Issue 1, (May 1975) 77-78, ISSN 0375-9601

Jeong, Y.; Sahu, J.; Payne, D.; \& Nilsson, J. (2004) Ytterbium-doped large-core fiber laser with $1.36 \mathrm{~kW}$ continuous-wave output power. Opt. Express, Vol. 12, Issue 25 (December 2004), 6088-6092, ISSN 1094-4087

Kawanaka, J.; Yamakawa, K.; Nishioka, H. \& Ueda, K. (2003) 30-mJ, diode-pumped, chirped-pulse Yb:YLF regenerative amplifier. Opt. Lett., Vol. 28, Issue 21 (November 2003), 2121-2123, ISSN 0146-9592

Kleinbauer, J.; Knappe, R.; \& Wallenstein, R. (2005) 13-W picoseconds Nd:GdVO 4 regenerative amplifier with $200-\mathrm{kHz}$ repetition rate. Appl. Phys. B, Vol. 81, No. 2-3 (July 2005), 163-166, ISSN 0946-2171

Kleinbauer, J.; Eckert, D.; Weiler, S.; \& Sutter, D. (2008) 80 W ultrafast CPA-free disk laser, Proc. SPIE, Vol. 6871 (February 2008), 68711B, ISSN 0277-786X

Koechner, W. (2006) Solid-State Laser Engineering, Springer, ISBN 978-0-387-29094-2, USA

Liu, C.; Riesbeck, T.; Wang, X.; Ge, J.; Xiang, Z.; Chen J. \& Eichler, H. (2008) Influence of spherical aberrations on the performance of dynamically stable resonators, Optics Communications, Vol. 281, Issue 20 (October, 2008) 5222-5228 ISSN 0030-4018.

Liu, H.; Gao, C.; Tao, J.; Zhao, W.; \& Wang, Y. (2008) Compact tunable high power picosecond source based on $\mathrm{Yb}$-doped fiber amplification of gain switch laser diode. Opt. Express, Vol. 16, Issue 11 (May 2008), 7888-7893, ISSN 1094-4087 
Lorenz, E. (1963) Deterministic nonperiodic flow. Journal of the Atmospheric Sciences, Vol. 20, Issue 2 (March 1963), 130-141, ISSN 0022-4928

Lowdermilk, W. \& Murray, J. (1980) The multipass amplifier: theory and numerical analysis. J. Appl. Phys., Vol. 51, No. 5 (May 1980), 2436-2444, ISSN 0021-8979

Magni, V. (1986) Resonators for solid-state lasers with large-volume fundamental mode and high alignment stability. Applied Optics, Vol. 25, No. 1 (January 1986), 107-118, ISSN 0003-6935

Matsushima, I.; Yashiro, H \& Tomie, T. (2006) $10 \mathrm{kHz} 40 \mathrm{~W}$ Ti:sapphire regenerative ring amplifier. Opt. Lett., Vol. 31, Issue 13 (July 2006), 2066-2068, ISSN 0146-9592

Meijer, J.; Dub, K.; Gillner, A.; Hoffmann, D.; Kovalenko, V.; Masuzawa, T.; Ostendorf, A.; Poprawe, R.; \& Schulz, W. (2002) Laser Machining by short and ultrashort pulses, state of the art and new opportunities in the age of the photons. CIRP Annals Manufacturing Technology, Vol. 51, Issue 2 (February 2002), 531-550, ISSN 0007-8506

Metzger, T.; Schwarz, A.; Teisset, C.; Sutter, D.; Killi, A.; Kienberger, R. \& Krausz, F. (2009) High-repetition-rate picosecond pump laser based on a Yb:YAG disk amplifier for optical parametric amplification. Opt. Lett., Vol. 34, No. 14 (July 2009), 2123-2125, ISSN 0146-9592

Mourou, G. \& Umstadter, D. (1992) Development and Applications of Compact HighIntensity Lasers. Phys. Fluids B, Vol. 4, No. 7 (July 1992), 2317-2325, ISSN 0899-8213

Müller, D.; Giesen, A \& Hügel, H. (2003) Picosecond thin-disk regenerative amplifier. Proceedings of SPIE, Vol. 5120 (November 2003), 281-286, ISSN 0277-786X

Murray, J. \& Lowdermilk, W. (1980) Nd:YAG regenerative amplifier. J. Appl. Phys., Vol. 51, No. 7 (July 1980), 3548-3555, ISSN 0021-8979

Nickel, D.; Stolzenburg, C.; Bevertt, A.; Geisen, A.; Haüssermann, J.; Butze, F.; \& Leitner, M. (2005) $200 \mathrm{kHz}$ electro-optic switch for ultrafast laser systems. Rev. Sci. Instrum., Vol. 76, No. 3 (March 2005), 033111-033111/7, ISSN 0034-6748

Norris, T. (1992) Femtosecond pulse amplification at $250 \mathrm{kHz}$ with a Ti:sapphire regenerative amplifier and application to continuum generation. Opt. Lett., Vol. 17, Issue. 14 (July 1992), 1009-1011, ISSN 0146-9592

Peterson, R.; Jenssen, H. \& Cassanho, A. (2002) Investigation of the spectroscopic properties of Nd:YVO $\mathrm{YV}_{4}$ In: Proc. OSA TOPS, Advanced Solid-State Lasers, M.E. Fermann and L.R. Marshall, (Ed.), Vol. 68, 294-298, OSA

Pugžlys, A.; Andriukaitis, G.; Baltuška, A.; Su, L.; Xu, J.; Li, H.; Li, R.; Lai, W.; Phua, P.; Marcinkevičius, A.; Fermann, M.; Giniūnas, L.; Danielius, R.; \& Ališauskas S. (2009) Multi-mJ, 200-fs, CW-pumped, cryogenically cooled, Yb,Na:CaF2 amplifier. Opt. Lett., Vol. 34, Issue 13 (July 2009), 2075-2077, ISSN 0146-9592

Raciukaitis, G.; Grishin, M.; Danielius, R.; Pocius, J. \& Giniūnas, L. (2006) High repetition rate ps- and fs- DPSS lasers for micromachining. Congress Proceedings (in CD), Vol. 99, Paper M1001, ISBN 0-912035-85-4, International Congress on Applications of Lasers \& Electro- Optics, ICALEO 2006, Scottsdale, USA, October 30- November 2, 2006, Laser Institute of America

Röser, F.; Eidam, T.; Rothhardt, J.; Schmidt, O.; Schimpf, D.; Limpert, J. \& Tünnermann, A. (2007) Millijoule pulse energy high repetition rate femtosecond fiber chirped-pulse amplification system. Opt. Lett., Vol. 32, Issue 12 (December 2007), 3495-3497, ISSN 0146-9592 
Ross, I.; New, G. \& Bates, P. (2007) Contrast limitation due to pump noise in an optical parametric chirped pulse amplification system. Optics Communications, Vol. 273, Issue 2 (May 2007), 510-514, ISSN 0030-4018

Sayinc, H.; Buenting, U.; Wandt, D.; Neumann, J. \& Kracht, D. (2009) Ultrafast high power Yb:KLuW regenerative amplifier. Opt. Express, Vol. 17, No. 17 (August 2009), 1506815071, ISSN 1094-4087

Siebold, M.; Hornung, M.; Hein, J.; Paunescu, G.; Sauerbrey, R.; Bergmann, T. \& Hollemann, G. (2004) A high-average-power diode-pumped $\mathrm{Nd}: \mathrm{YVO}_{4}$ regenerative laser amplifier for picosecond-pulses. Applied Physics B, Vol. 78, Num. 3-4 (February 2004), 287-290, ISSN 0946-2171

Siebold, M.; Hein, J.; Hornung, M.; Podleska, S.; Kaluza, M.; Bock, S. \& Sauerbrey, R. (2008) Diode-pumped lasers for ultra-high peak power. Appl. Phys. B, Vol. 90, No. 3-4 (March 2008), 431-437, ISSN 0946-2171

Speiser, J. \& Giesen, A. (2008) Scaling of thin disk pulse amplifiers. Proc. SPIE, Vol. 6871 (February 2008), 68710J, ISSN 0277-786X

Strickland, D. \& Mourou, G. (1985) Compression of amplified chirped optical pulses. Opt. Comm., Vol. 56, Issue 3 (March 1985), 219-221, ISSN 0030-4018.

Svelto, O. (1998) Principles of Lasers, Plenum Press, ISBN 0-306-45748-2, New-York

Tang, D.; Ng, S.; Qin, L.; \& Meng, X. (2003) Deterministic chaos in a diode-pumped Nd:YAG laser passively Q switched by a $\mathrm{Cr}^{4+}$ YAG crystal. Opt. Lett., Vol. 28, Issue 5 (March 2003), 325-327, ISSN 0146-9592

Valling, S.; Fordell, T. \& Lindberg, A. (2005) Experimental and numerical intensity time series of an optically injected solid state laser. Opt. Commun., Vol. 254, Issue 4-6 (October 2005), 282-289, ISSN 0030-4018

Walker, B.; Toth, C.; Fittinghoff, D.; Guo, T.; Kim, D.; Rose-Petruck, C.; Squier, J.; Yamakawa, K.; Wilson, K.; \& Barty, B. (1999) A $50 \mathrm{EW} / \mathrm{cm}^{2}$ Ti:sapphire laser system for studying relativistic light-matter interactions. Opt. Express, Vol. 5, Issue 10 (November 1999), 196-202, ISSN 1094-4087 


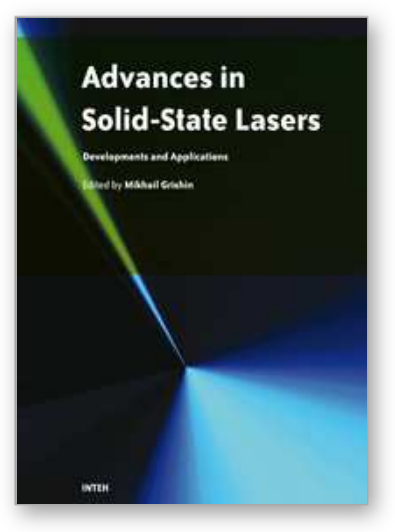

\author{
Advances in Solid State Lasers Development and Applications \\ Edited by Mikhail Grishin
}

ISBN 978-953-7619-80-0

Hard cover, 630 pages

Publisher InTech

Published online 01, February, 2010

Published in print edition February, 2010

Invention of the solid-state laser has initiated the beginning of the laser era. Performance of solid-state lasers improved amazingly during five decades. Nowadays, solid-state lasers remain one of the most rapidly developing branches of laser science and become an increasingly important tool for modern technology. This book represents a selection of chapters exhibiting various investigation directions in the field of solid-state lasers and the cutting edge of related applications. The materials are contributed by leading researchers and each chapter represents a comprehensive study reflecting advances in modern laser physics. Considered topics are intended to meet the needs of both specialists in laser system design and those who use laser techniques in fundamental science and applied research. This book is the result of efforts of experts from different countries. I would like to acknowledge the authors for their contribution to the book. I also wish to acknowledge Vedran Kordic for indispensable technical assistance in the book preparation and publishing.

\title{
How to reference
}

In order to correctly reference this scholarly work, feel free to copy and paste the following:

Mikhail Grishin and Andrejus Michailovas (2010). Dynamics of Continuously Pumped Solid-State Regenerative Amplifiers, Advances in Solid State Lasers Development and Applications, Mikhail Grishin (Ed.), ISBN: 978953-7619-80-0, InTech, Available from: http://www.intechopen.com/books/advances-in-solid-state-lasersdevelopment-and-applications/dynamics-of-continuously-pumped-solid-state-regenerative-amplifiers

\section{INTECH}

open science | open minds

\section{InTech Europe}

University Campus STeP Ri

Slavka Krautzeka 83/A

51000 Rijeka, Croatia

Phone: +385 (51) 770447

Fax: +385 (51) 686166

www.intechopen.com

\section{InTech China}

Unit 405, Office Block, Hotel Equatorial Shanghai

No.65, Yan An Road (West), Shanghai, 200040, China

中国上海市延安西路65号上海国际贵都大饭店办公楼 405 单元

Phone: +86-21-62489820

Fax: $+86-21-62489821$ 
(C) 2010 The Author(s). Licensee IntechOpen. This chapter is distributed under the terms of the Creative Commons Attribution-NonCommercialShareAlike-3.0 License, which permits use, distribution and reproduction for non-commercial purposes, provided the original is properly cited and derivative works building on this content are distributed under the same license. 